quatrième série-tome $46 \quad$ fascicule 5 septembre-octobre 2013

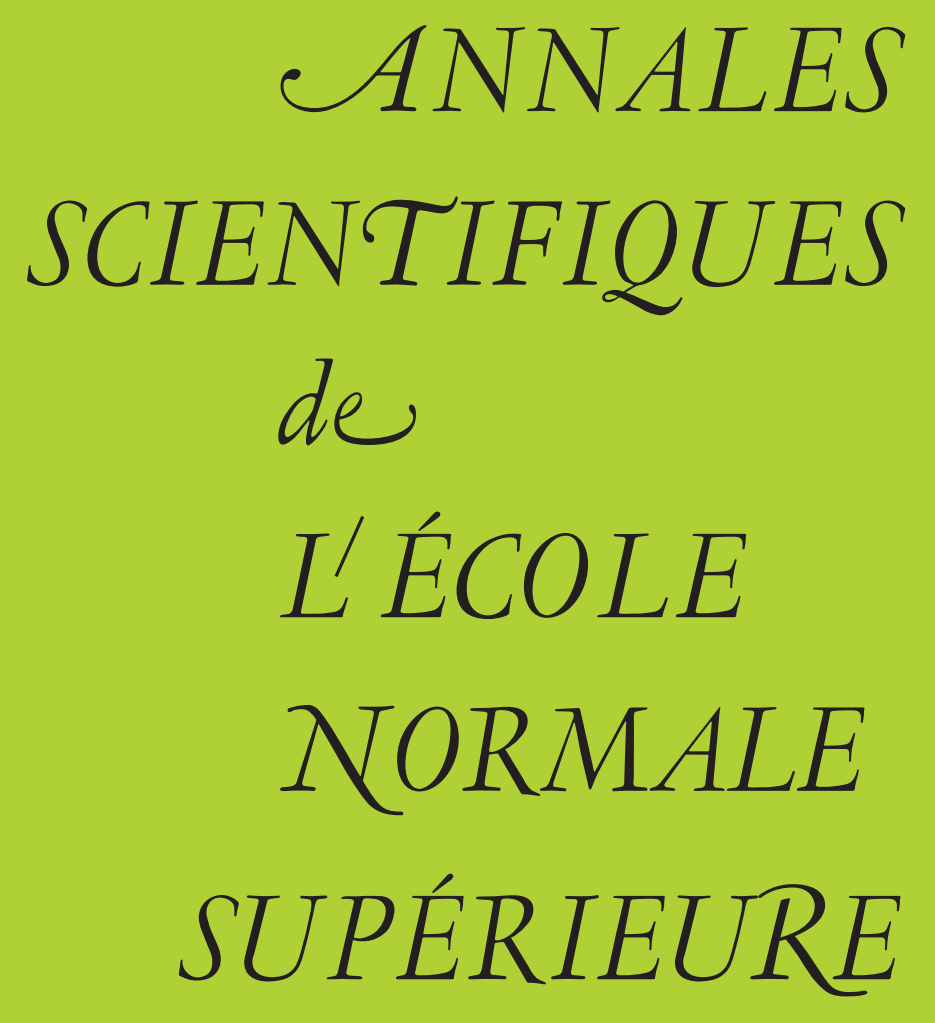

Laurent CHARLES \& Álvaro PELAYO \& San VŨ NGỌC Isospectrality for quantum toric integrable systems 


\title{
ISOSPECTRALITY FOR QUANTUM TORIC INTEGRABLE SYSTEMS
}

\author{
By Laurent CHARleS, Álvaro PElayo and San VŨ NGỌC
}

To Peter Sarnak on his sixtieth birthday, with admiration

\begin{abstract}
We give a full description of the semiclassical spectral theory of quantum toric integrable systems using microlocal analysis for Toeplitz operators. This allows us to settle affirmatively the isospectral problem for quantum toric integrable systems: the semiclassical joint spectrum of the system, given by a sequence of commuting Toeplitz operators on a sequence of Hilbert spaces, determines the classical integrable system given by the symplectic manifold and commuting Hamiltonians. This type of problem belongs to the realm of classical questions in spectral theory going back to pioneer works of Colin de Verdière, Guillemin, Sternberg and others in the 1970s and 1980s.

RÉsumé. - Nous donnons une description complète du spectre de tout système intégrable torique quantique, au moyen de l'analyse microlocale des opérateurs de Toeplitz. Ceci résout la question de l'isospectralité pour cette classe de systèmes intégrables : le spectre semi-classique d'un système intégrable quantique torique détermine le système intégrable classique sous-jacent à symplectomorphisme près. Nous donnons aussi une description complète de la théorie spectrale semi-classique des systèmes intégrables toriques quantiques. Ces questions sont classiques en théorie spectrale et remontent aux travaux fondateurs de Colin de Verdière, Guillemin et Sternberg parmi d'autres dans les années 70 et 80 .
\end{abstract}

\section{Introduction}

This paper gives a full description of the semiclassical spectral theory of quantum toric integrable systems in any finite dimension. The classical limits corresponding to quantum toric integrable systems are the so called symplectic toric manifolds or toric systems. Such a system consists of a compact symplectic $2 n$-manifold equipped with $n$ commuting Hamiltonians $f_{1}, \ldots, f_{n}$ with periodic flows. The paper combines geometric techniques from the theory of toric manifolds, in the complex-algebraic and symplectic settings, with recently developed microlocal analytic methods for Toeplitz operators. 
As a consequence of the spectral theory we develop, we answer the isospectrality question for quantum toric integrable systems, in any finite dimension: the semiclassical joint spectrum of a quantum toric integrable system, given by a sequence of commuting Toeplitz operators acting on quantum Hilbert spaces, determines the classical system given by the symplectic manifold and Poisson commuting functions, up to symplectic isomorphisms. This type of symplectic isospectral problem belongs to the realm of classical questions in inverse spectral theory and microlocal analysis, going back to pioneer works of Colin de Verdière $[14,13]$ and Guillemin-Sternberg [37] in the 1970s and 1980s. Colin de Verdière's works are an important inspiration for the present paper.

The question of isospectrality in Riemannian geometry may be traced back to Weyl [72, 73] and is most well known thanks to Kac's article [41], who himself attributes the question to Bochner. Kac popularized the sentence: "can one hear the shape of a drum?," to refer to this type of isospectral problem. The spectral theory developed in this paper exemplifies a striking difference with Riemannian geometry, where this type of isospectrality rarely holds true, and suggests that symplectic invariants are much better encoded in spectral theory than Riemannian invariants. An approach to this problem for general integrable systems is suggested in the last two authors' article [60]. We refer to Section 8 for further remarks, and references, in these directions.

\section{Joint spectrum}

In order to state our results, let us introduce the required terminology. If $(M, \omega)$ is a symplectic manifold, a smooth map $\mu=\left(\mu_{1}, \ldots, \mu_{n}\right): M \rightarrow \mathbb{R}^{n}$ is called a momentum map for a Hamiltonian $n$-torus action on $M$ if the Hamiltonian flows $t_{j} \mapsto \varphi_{\mu_{j}}^{t_{j}}$ are periodic of period 1, and pairwise commute :

$$
\varphi_{\mu_{j}}^{t_{j}} \circ \varphi_{\mu_{i}}^{t_{i}}=\varphi_{\mu_{i}}^{t_{i}} \circ \varphi_{\mu_{j}}^{t_{j}},
$$

so that they define an action of $\mathbb{R}^{n} / \mathbb{Z}^{n}$. If this action is effective and $M$ is compact, $2 n$-dimensional and connected, we call $(M, \omega, \mu)$ a symplectic toric manifold.

By the Atiyah and Guillemin-Sternberg theorem, for any torus Hamiltonian action on a connected compact manifold, the image of the momentum map is a rational convex polytope [1,36]. For a symplectic toric manifold, the momentum polytope $\Delta \subset \mathbb{R}^{n}$ has the additional property that for each vertex $v$ of $\Delta$, the primitive normal vectors to the facets meeting at $v$ form a basis of the integral lattice $\mathbb{Z}^{n}$. We call such a polytope a Delzant polytope.

A now standard procedure introduced by B. Kostant [43, 44, 45, 46] and J.-M. Souriau $[62,63]$ to quantize a symplectic compact manifold $(M, \omega)$ is to introduce a prequantum bundle $\mathcal{L} \rightarrow M$, that is a Hermitian line bundle with curvature $\frac{1}{\mathrm{i}} \omega$ and a complex structure $j$ compatible with $\omega$. One then defines the quantum space as the space

$$
\mathcal{H}_{k}:=\mathrm{H}^{0}\left(M, \mathscr{L}^{k}\right)
$$

of holomorphic sections of $\mathcal{L}^{k}$. The parameter $k$ is a positive integer, the semiclassical limit corresponds to the large $k$ limit. A description of this procedure, which is called geometric quantization, is given by Kostant and Pelayo in [47] from the angle of Lie theory and representation theory.

4 e SÉRIE - TOME $46-2013$ - No 5 
Not all symplectic manifolds have a complex structure or a prequantum bundle. However a symplectic toric manifold always admits a compatible complex structure, which is not unique. Furthermore a symplectic toric manifold $M$ with momentum map $\mu: M \rightarrow \mathbb{R}^{n}$ is prequantizable if and only if there exists $c \in \mathbb{R}^{n}$ such that the vertices of the polytope $\mu(M)+c$ belong to $2 \pi \mathbb{Z}^{n}$ (see Section 3). If it is the case, the prequantum bundle is unique up to isomorphisms.

In many papers, a prequantum bundle is defined as a line bundle with curvature $\frac{1}{2 \pi \mathrm{i}} \omega$. With this normalization, the cohomology class of $\omega$ is integral and the prequantization condition for toric manifolds is that, up to translation, the momentum polytope has integral vertices. This normalization may look simpler than ours, which includes a $2 \pi$-factor. Nevertheless, our choice is justified by the Weyl law. Indeed, with our normalization, the dimension of the quantum space $\mathcal{H}_{k}$ is

$$
\left(\frac{k}{2 \pi}\right)^{n} \operatorname{vol}(M, \omega)+\theta\left(k^{n-1}\right) .
$$

Associated to such a quantization there is an algebra $\mathscr{T}(M, \mathcal{L}, j)$ of operators

$$
T=\left(T_{k}: \mathscr{H}_{k} \rightarrow \mathscr{H}_{k}\right)_{k \in \mathbb{N}^{*}}
$$

called Toeplitz operators. This algebra plays the same role as the algebra of semiclassical pseudodifferential operators for a cotangent phase space. Here the semiclassical parameter is $\hbar=1 / k$. A Toeplitz operator has a principal symbol, which is a smooth function on the phase space $M$. If $T$ and $S$ are Toeplitz operators, then $\left(T_{k}+k^{-1} S_{k}\right)_{k \in \mathbb{N}^{*}}$ is a Toeplitz operator with the same principal symbol as $T$. If $T_{k}$ is Hermitian (i.e., self-adjoint) for $k$ sufficiently large, then the principal symbol of $T$ is real-valued. Two Toeplitz operators $\left(T_{k}\right)_{k \in \mathbb{N}^{*}}$ and $\left(S_{k}\right)_{k \in \mathbb{N}^{*}}$ commute if $T_{k}$ and $S_{k}$ commute for every $k$.

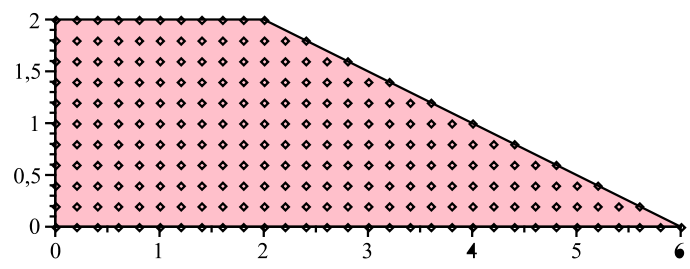

FiguRE 1. "Model image" of the spectrum of a normalized quantum toric integrable system.

We shall also need the following definitions. If $P_{1}, \ldots, P_{n}$ are mutually commuting endomorphisms of a finite dimensional vector space, then the joint spectrum of $P_{1}, \ldots, P_{n}$ is the set of $\left(\lambda_{1}, \ldots, \lambda_{n}\right) \in \mathbb{C}^{n}$ such that there exists a non-zero vector $v$ for which

$$
P_{j} v=\lambda_{j} v,
$$

for all $j=1, \ldots, n$. It is denoted by $\operatorname{JointSpec}\left(P_{1}, \ldots, P_{n}\right)$. The Hausdorff distance between two subsets $A$ and $B$ of $\mathbb{R}^{n}$ is

$$
\mathrm{d}_{H}(A, B):=\inf \left\{\epsilon>0 \mid A \subseteq B_{\epsilon} \text { and } B \subseteq A_{\epsilon}\right\},
$$


where for any subset $X$ of $\mathbb{R}^{n}$, the set $X_{\epsilon}$ is $X_{\epsilon}:=\bigcup_{x \in X}\left\{m \in \mathbb{R}^{n} \mid\|x-m\| \leqslant \epsilon\right\}$. If $\left(A_{k}\right)_{k \in \mathbb{N}^{*}}$ and $\left(B_{k}\right)_{k \in \mathbb{N}^{*}}$ are sequences of subsets of $\mathbb{R}^{n}$, we say that

$$
A_{k}=B_{k}+\Theta\left(k^{-\infty}\right)
$$

if $\mathrm{d}_{H}\left(A_{k}, B_{k}\right)=\theta\left(k^{-N}\right)$ for all $N \in \mathbb{N}^{*}$.

Our main result describes in full the joint spectrum of a quantum toric integrable system.

Theorem 1.1 (Joint Spectral Theorem). - Let $\left(M, \omega, \mu: M \rightarrow \mathbb{R}^{n}\right)$ be a symplectic toric manifold equipped with a prequantum bundle $\mathcal{L}$ and a compatible complex structure $j$. Let $T_{1}, \ldots, T_{n}$ be commuting Toeplitz operators of $\mathscr{T}(M, \mathcal{L}, j)$ whose principal symbols are the components of $\mu$. Then the joint spectrum of $T_{1}, \ldots, T_{n}$ satisfies

$$
\text { JointSpec }\left(T_{1}, \ldots, T_{n}\right)=g\left(\Delta \cap\left(v+\frac{2 \pi}{k} \mathbb{Z}^{n}\right) ; k\right)+\vartheta\left(k^{-\infty}\right)
$$

where $\Delta=\mu(M)$, $v$ is any vertex of $\Delta$ and $g(\cdot ; k): \mathbb{R}^{n} \rightarrow \mathbb{R}^{n}$ admits a $\mathrm{C}^{\infty}$-asymptotic expansion of the form

$$
g(\cdot ; k)=\mathrm{Id}+k^{-1} g_{1}+k^{-2} g_{2}+\cdots
$$

where each $g_{j}: \mathbb{R}^{n} \rightarrow \mathbb{R}^{n}$ is smooth. Moreover, for all sufficiently large $k$, the multiplicity of the eigenvalues of $\operatorname{Joint} \operatorname{Spec}\left(T_{1}, \ldots, T_{n}\right)$ is 1 , and there exists a small constant $\delta>0$ such that each ball of radius $\frac{\delta}{k}$ centered at an eigenvalue contains precisely only that eigenvalue.

Thus the joint spectrum of a quantum toric integrable system can be obtained by taking the $k^{-1} \mathbb{Z}^{n}$ lattice points in a polytope $\Delta$ (as in Figure 1), and applying a small smooth deformation $g$ (as in Figure 2).

\section{Isospectrality}

We present next the isospectral theorem for toric systems. An easy consequence of the previous theorem is that the momentum polytope $\Delta$ is the Hausdorff limit of the joint spectrum of the quantum system, that is $\Delta$, consists of the $\lambda \in \mathbb{R}^{n}$ such that for any neighborhood $U$ of $\lambda, U \cap \operatorname{JointSpec}\left(T_{1, k}, \ldots, T_{n, k}\right) \neq \varnothing$ when $k$ is sufficiently large.

Recall that two symplectic toric manifolds $(M, \omega, \mu)$ and $\left(M^{\prime}, \omega^{\prime}, \mu^{\prime}\right)$ are isomorphic if there exists a symplectomorphism $\varphi: M \rightarrow M^{\prime}$ such that

$$
\varphi^{*} \mu^{\prime}=\mu \text {. }
$$

By the Delzant classification theorem [21], a symplectic toric manifold is determined up to isomorphism by its momentum polytope. Furthermore, for any Delzant polytope $\Delta$, Delzant constructed in [21] a symplectic toric manifold $\left(M_{\Delta}, \omega_{\Delta}, \mu_{\Delta}\right)$ with momentum polytope $\Delta$. Now we are ready to state our isospectral theorem (see Figure 2 for an illustration of the semiclassical joint spectrum).

Corollary 1.2 (Isospectral Theorem). - Let $\left(M, \omega, \mu: M \rightarrow \mathbb{R}^{n}\right)$ be a symplectic toric manifold equipped with a prequantum bundle $\mathcal{L}$ and a compatible complex structure $j$. Let $T_{1}, \ldots, T_{n}$ be commuting Toeplitz operators of $\mathscr{T}(M, \mathcal{L}, j)$ whose principal symbols are the components of $\mu$. Then

$$
\Delta:=\lim _{k \rightarrow \infty} \operatorname{JointSpec}\left(T_{1}, \ldots, T_{n}\right)
$$

$4^{\text {e }}$ SÉRIE - TOME $46-2013-$ NN$^{\circ} 5$ 

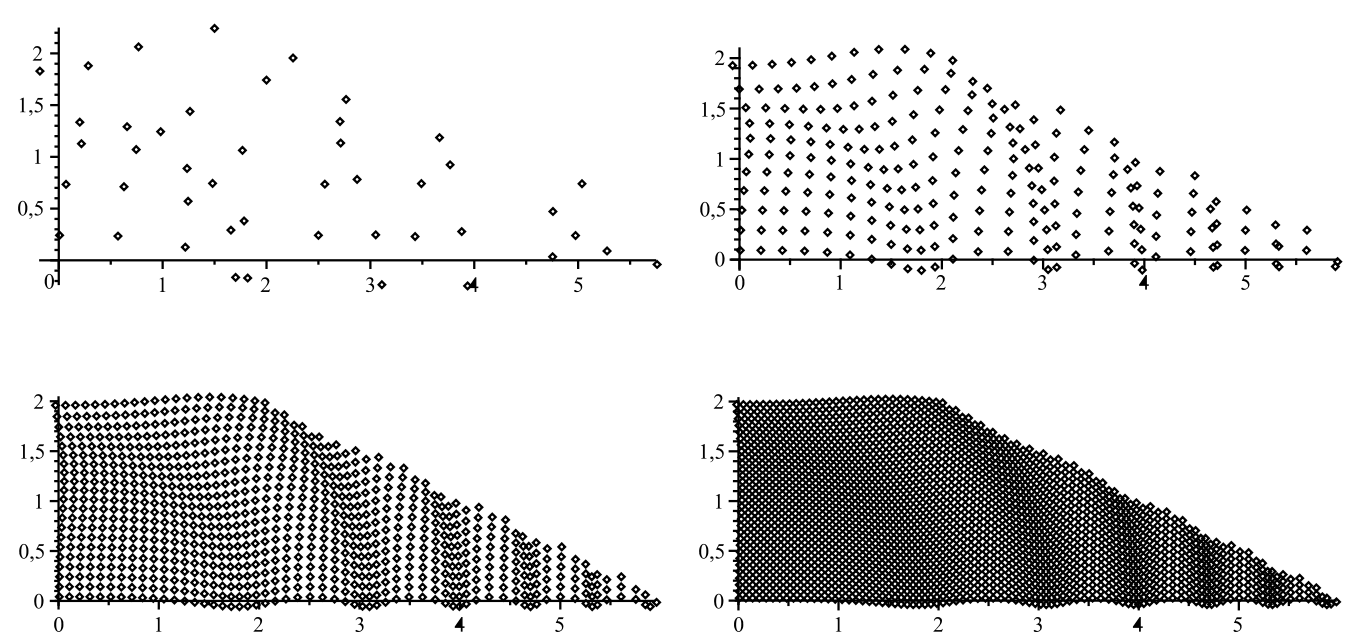

FIgURE 2. Sequence of images of the spectra of a quantum toric integrable systems as the semiclassical parameter $\hbar$ goes to 0 . The spectra lie on a plane, so they correspond to a four-dimensional integrable system with two degrees of freedom. In the Hausdorff limit corresponding to $\hbar=0$, the spectra converge to a polytope; this is proved for a general quantum toric system in any dimension by Corollary 1.2. Therefore, one can recover the classical system from the semiclassical spectrum (i.e., the spectrum of the family of Toeplitz operators as $\hbar$ approaches 0 ).

is a Delzant polytope and $(M, \omega, \mu)$ is isomorphic with $\left(M_{\Delta}, \omega_{\Delta}, \mu_{\Delta}\right)$. In other words, one can recover the classical system from the limit of the joint spectrum.

This type of inverse result is classical and belongs to the realm of questions in inverse spectral theory, going back to similar questions raised (and in many cases answered) by pioneer works of Colin Verdière and Guillemin-Sternberg in the 1970s and 1980s. Many other contributions followed their works, for instance Datchev-Hezari-Ventura [20] and Iantchenko-Sjöstrand-Zworski [40]. A few global spectral results have also been obtained recently, for instance by Vũ Ngọc [71] for one degree of freedom pseudodifferential operators, or in the article by Dryden, Guillemin, and Sena-Dias [22] in which an equivariant spectrum of the Laplace operator is considered, and the references therein. See Section 8 for further references.

\section{Metaplectic correction}

Introducing a metaplectic correction refers to twisting the prequantum bundle (or its powers) by a half-form bundle. The metaplectic correction allows to obtain an easier control of the subprincipal terms in the semiclassical limit. In the following theorem we improve the previous Joint spectral Theorem by giving the explicit description of the spectrum up to $\theta\left(k^{-2}\right)$.

Recall that a half-form bundle of a complex manifold is a square root of its canonical bundle. Given a symplectic manifold $(M, \omega)$ with a compatible complex structure, a prequantum bundle $\mathcal{L}$ and a half-form bundle $\delta$, the associated quantum space is $\mathcal{H}_{\mathrm{m}, k}=\mathrm{H}^{0}\left(M, \mathcal{L}^{k} \otimes \delta\right)$. 
We can define Toeplitz operators in this setting together with their principal symbols. To state our result, we also need the notion of subprincipal symbol of a Toeplitz operator whose definition is recalled in Section 5. Two Toeplitz operators with the same principal symbol are equal up to $\Theta\left(k^{-2}\right)$ if and only if they have the same subprincipal symbols.

As we will see in Section 7, a symplectic toric manifold with moment polytope $\Delta \subset \mathbb{R}^{n}$ has a half-form bundle if and only if there exists a vector $u \in \mathbb{Z}^{n}$ such that for any onecodimensional face $f$ of $\Delta$, the scalar product of a primitive normal of $f$ with $u$ is odd. Such a vector, if it exists, is uniquely determined modulo $(2 \mathbb{Z})^{n}$. We denote it by $u_{\Delta}$.

Theorem 1.3 (Joint Spectral Theorem with metaplectic correction)

Let $\left(M, \omega, \mu: M \rightarrow \mathbb{R}^{n}\right)$ be a symplectic toric manifold equipped with a prequantum bundle $\mathcal{L}$, a compatible complex structure $j$ and a half-form bundle $\delta$. Let $T_{1}, \ldots, T_{n}$ be commuting Toeplitz operators of $\mathcal{H}_{\mathrm{m}, k}$ whose principal symbols are the components of $\mu$. Then the joint spectrum of $T_{1}, \ldots, T_{n}$ satisfies

$$
\operatorname{JointSpec}\left(T_{1}, \ldots, T_{n}\right)=g\left(\Delta \cap\left(v+\frac{2 \pi}{k}\left(\mathbb{Z}^{n}+u_{\Delta} / 2\right)\right) ; k\right)+\vartheta\left(k^{-\infty}\right)
$$

where $\Delta=\mu(M)$, $v$ is any vertex of $\Delta$ and $g(\cdot ; k): \mathbb{R}^{n} \rightarrow \mathbb{R}^{n}$ admits a $\mathrm{C}^{\infty}$-asymptotic expansion of the form

$$
g(\cdot ; k)=\mathrm{Id}+k^{-1} g_{1}+k^{-2} g_{2}+\cdots
$$

where each $g_{j}: \mathbb{R}^{n} \rightarrow \mathbb{R}^{n}$ is smooth. Furthermore $g_{1}$ is determined by

$$
g_{1}^{i}(E)=\int_{0}^{1} f_{1}^{i}\left(\varphi_{\mu^{i}}^{t}(x)\right) \mathrm{d} t, \quad \text { for all } E \in \Delta, x \in \mu^{-1}(E)
$$

where $i=1, \ldots, n, f_{1}^{i}$ is the subprincipal symbol of $T^{i}$ and $\varphi_{\mu^{i}}^{t}$ is the Hamiltonian flow of $\mu^{i}$. Moreover, for all sufficiently large $k$, the multiplicity of the eigenvalues of $\operatorname{Joint} \operatorname{Spec}\left(T_{1}, \ldots, T_{n}\right)$ $i$ is 1 , and there exists a small constant $\delta>0$ such that each ball of radius $\frac{\delta}{k}$ centered at an eigenvalue contains precisely only that eigenvalue.

Besides the average of the subprincipal symbols, it is interesting to note the shift by $u_{\Delta} / 2$ so that no eigenvalue lies on the boundary of $g(\Delta)$ when $k$ is sufficiently large, cf. Figure 5 for the spectrum of a model toric system with metaplectic correction.

\section{Toeplitz quantization}

A natural question is how to decide whether a given integrable system can be quantized. A discussion of this problem may be found in Garay-Van Straten [31] and the references therein (they work with pseudodifferential operators, instead of Toeplitz operators). Concretely, given a prequantizable symplectic manifold endowed with an integrable system $\left(f_{1}, \ldots, f_{n}\right)$, it may not be possible to find a set of commuting Toeplitz operators whose principal symbols are $f_{1}, \ldots, f_{n}$, respectively. However, in the case of toric integrable systems, we will obtain, as a byproduct of the proof of Theorem 1.1, the following existence result.

Theorem 1.4 (Existence of Toeplitz quantization). - Let $\left(M, \omega, \mu: M \rightarrow \mathbb{R}^{n}\right)$ be a symplectic toric manifold equipped with a prequantum bundle $\mathcal{L}$ and a compatible complex

4 e SÉRIE - TOME $46-2013$ - No 5 
structure $j$. Then there exist mutually commuting Toeplitz operators $T_{1}, \ldots, T_{n}$ in $\mathscr{T}(M, \mathcal{L}, j)$ whose principal symbols are the components of $\mu$.

The proofs in the paper combine geometric ideas from the theory of toric manifolds in the complex and symplectic settings with microlocal analytic methods dealing with semiclassical Toeplitz operators that were developed by the first author [8, 7, 9, 11].

\section{Model for a symplectic toric manifold}

We review the ingredients from the theory of symplectic toric manifolds which we need for this paper, namely the Delzant construction.

This section gives a fast review of the necessary background to read this paper.

\section{Terminology from group actions}

Let $(M, \omega)$ be a symplectic manifold, i.e., the pair consisting of a smooth manifold $M$ and a symplectic form $\omega$ on $M$, that is, a non-degenerate differential closed 2-form on $M$.

Suppose that a Lie group $G$ acts on a symplectic manifold $(M, \omega)$ symplectically, i.e., by diffeomorphisms which preserve the symplectic form. We denote by $(t, p) \mapsto t \cdot p$ the action $G \times M \rightarrow M$ of $G$ on $M$. Let $\mathfrak{g}$ be the Lie algebra of $G$. Any element $X \in \mathfrak{g}$ generates a vector field $X_{M}$ on $M$, called the infinitesimal generator, given by

$$
X_{M}(p):=\left.\frac{\mathrm{d}}{\mathrm{d} t}\right|_{t=0} \exp (t X) \cdot p
$$

where exp: $\mathfrak{g} \rightarrow G$ is the exponential map of Lie theory. As usual, we write $\iota_{X_{M}} \omega:=$ $\omega\left(X_{M}, \cdot\right) \in \Omega^{1}(M)$ for the contraction 1-form. The $G$-action on $(M, \omega)$ is said to be Hamiltonian if there exists a smooth invariant map $\mu: M \rightarrow \mathfrak{g}^{*}$, called the momentum map, such that for all $X \in \mathfrak{g}$ we have that

$$
\iota_{X_{M}} \omega=-\mathrm{d}\langle\mu, X\rangle,
$$

where $\langle\cdot, \cdot\rangle: \mathfrak{g}^{*} \times \mathfrak{g} \rightarrow \mathbb{R}$ is the duality pairing. If the first de Rham cohomology group $\mathrm{H}_{\mathrm{dR}}^{1}(M)$ is trivial, then any symplectic $T$-action on $M$ is Hamiltonian.

The $G$-action is effective if the intersection of all stabilizer subgroups

$$
G_{p}:=\{g \in G \mid g \cdot p=p\}, p \in M,
$$

is the trivial group. The $G$-action is free if $G_{p}$ is the trivial group for all points $p \in M$.

ExAmple 2.1. - The simplest example of a Hamiltonian group action is given by the standard symplectic sphere $S^{2}$ with the rotational $S^{1}$-action. It is easy to check that the momentum map for this action is the height function $\mu(\theta, h)=h$. See Figure 3 .

We will need the following classical result. Recall that a torus $T$ is a compact, connected, commutative Lie group. As a Lie group, $T$ is isomorphic to a finite product of circles $S^{1}$. 
Theorem 2.2 (Atiyah [1], Guillemin and Sternberg [36]). - If a torus T with Lie algebrat acts on a compact, connected $2 n$-dimensional symplectic manifold $(M, \omega)$ in a Hamiltonian fashion, then the image $\mu(M)$ under the momentum map $\mu: M \rightarrow \mathfrak{t}^{*}$ of the action is a convex polytope $\Delta \subset \mathfrak{t}^{*}$.

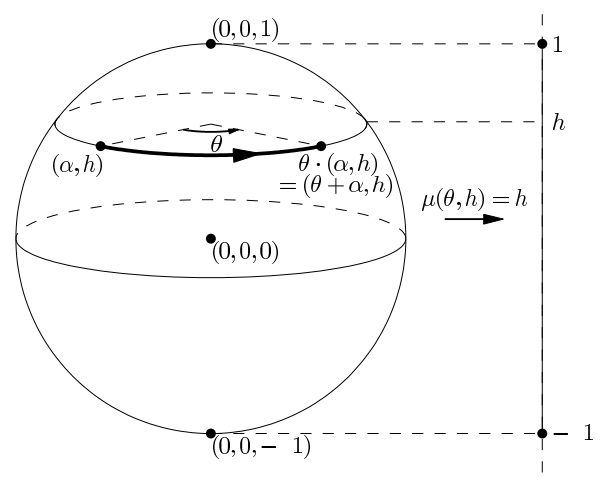

FIGURE 3. The momentum map for the 2 -sphere $S^{2}$ is the height function $\mu(\theta, h)=h$. The image of $S^{2}$ under the momentum map $\mu$ is the closed interval $\Delta:=[-1,1]$. Note that as predicted by the Atiyah-Guillemin-Sternberg Theorem, the interval $[-1,1]$ is equal to the image under $\mu$ of the set $\{(0,0,-1),(0,0,1)\}$ of fixed points of the Hamiltonian $S^{1}$-action on $S^{2}$ by rotations about the vertical axis.

\section{Delzant construction of toric systems}

Let $T$ be an $n$-dimensional torus. Denote by $\mathfrak{t}$ the Lie algebra of $T$ and by $\mathfrak{t}_{\mathbb{Z}}$ the kernel of the exponential map exp $: \mathfrak{t} \rightarrow T$. We denote the isomorphism $\mathfrak{t} / \mathfrak{t}_{\mathbb{Z}} \rightarrow T$ also by exp. A symplectic toric manifold $\left(M, \omega, T, \mu: M \rightarrow \mathfrak{t}^{*}\right)$ is a symplectic compact connected manifold $(M, \omega)$ of dimension $2 n$ with an effective Hamiltonian action of $T$ with momentum map $\mu$.

When $T=\mathbb{R}^{n} / \mathbb{Z}^{n}$ so that $\mathfrak{t} \simeq \mathbb{R}^{n}$, we recover the definition given in the introduction. Two symplectic toric manifolds $(M, \omega, T, \mu)$ and $\left(M^{\prime}, \omega^{\prime}, T, \mu^{\prime}\right)$ are isomorphic if there exists a symplectomorphism $\varphi: M \rightarrow M^{\prime}$ such that $\mu^{\prime} \circ \varphi=\mu$. If it is the case, $\varphi$ intertwines the torus actions.

We present the construction of Delzant [21] of symplectic toric manifolds as reduced phase spaces.

Step 1 (Starting from a Delzant polytope $\Delta \subset \mathfrak{t}^{*}$ ). - Let $\Delta$ be an $n$-dimensional convex polytope in the dual Lie algebra $\mathfrak{t}^{*}$. We denote by $F$ and $V$ the set of all codimension one faces and vertices of $\Delta$, respectively. Every face of $\Delta$ is compact. For every $v \in V$, we write $F_{v}=\{f \in F \mid v \in f\}$. The polytope $\Delta$ is called a Delzant polytope if it has the following properties, see Guillemin [34, p. 8].

i) For each $f \in F$ there exist $X_{f} \in \mathfrak{t}_{\mathbb{Z}}$ and $\lambda_{f} \in \mathbb{R}$ such that the hyperplane which contains $f$ is equal to the set of all $\xi \in \mathfrak{t}^{*}$ such that $\left\langle X_{f}, \xi\right\rangle+\lambda_{f}=0$, and $\Delta$ is contained in the set of all $\xi \in \mathfrak{t}^{*}$ such that $\left\langle X_{f}, \xi\right\rangle+\lambda_{f} \geqslant 0$. 
Note: The vector $X_{f}$ and constant $\lambda_{f}$ are made unique by requiring that they are not an integral multiple of another such vector and constant, respectively.

ii) For every vertex $v \in V$, the vectors $X_{f}$ with $f \in F_{v}$ form a $\mathbb{Z}$-basis of the integral lattice $\mathfrak{t}_{\mathbb{Z}}$ in $\mathfrak{t}$.

It follows that

$$
\Delta=\left\{\xi \in \mathfrak{t}^{*} \mid\left\langle X_{f}, \xi\right\rangle+\lambda_{f} \geqslant 0 \text { for every } f \in F\right\} .
$$

Also, $\#\left(F_{v}\right)=n$ for every $v \in V$.

Step 2 (The epimorphism $\mathbb{R}^{F} / \mathbb{Z}^{F} \rightarrow T$ and the subtorus $N$ ). - Let $\pi: \mathbb{R}^{F} \rightarrow \mathfrak{t}$ be defined by

$$
\pi(t):=\sum_{f \in F} t_{f} X_{f}, \quad t \in \mathbb{R}^{F} .
$$

Because, for any vertex $v$, the $X_{f}$ with $f \in F_{v}$ form a $\mathbb{Z}$-basis of $\mathfrak{t}_{\mathbb{Z}}$, we have $\pi\left(\mathbb{Z}^{F}\right)=\mathfrak{t}_{\mathbb{Z}}$ and $\pi\left(\mathbb{R}^{F}\right)=\mathfrak{t}$. It follows that $\pi$ induces an epimorphism

$$
\pi^{\prime}: \mathbb{R}^{F} / \mathbb{Z}^{F}=(\mathbb{R} / \mathbb{Z})^{F} \rightarrow \mathfrak{t} / \mathfrak{t}_{\mathbb{Z}}
$$

and we have the corresponding epimorphism $\exp \circ \pi^{\prime}: \mathbb{R}^{F} / \mathbb{Z}^{F} \rightarrow T$.

Write $\mathfrak{n}:=\operatorname{ker} \pi$, a linear subspace of $\mathbb{R}^{F}$, and

$$
N:=\operatorname{ker}\left(\exp \circ \pi^{\prime}\right) \subseteq \mathbb{R}^{F} / \mathbb{Z}^{F},
$$

a compact commutative subgroup of the torus $\mathbb{R}^{F} / \mathbb{Z}^{F}$. One can check that $N$ is connected, and therefore isomorphic to $\mathfrak{n} / \mathfrak{n}_{\mathbb{Z}}$, where $\mathfrak{n}_{\mathbb{Z}}:=\mathfrak{n} \cap \mathbb{Z}^{F}$ is the integral lattice in $\mathfrak{n}$ of the torus $N$.

Step 3 (Action of $N$ on $\mathbb{C}^{F}$ ). - On the complex vector space $\mathbb{C}^{F}$, we have the action of the torus $\mathbb{R}^{F} / \mathbb{Z}^{F}$, where $t \in \mathbb{R}^{F} / \mathbb{Z}^{F}$ maps $z \in \mathbb{C}^{F}$ to the element $t \cdot z \in \mathbb{C}^{F}$ defined by

$$
(t \cdot z)_{f}=\mathrm{e}^{2 \pi \mathrm{i} t_{f}} z_{f}, \quad f \in F .
$$

This action is Hamiltonian with momentum $\mu: \mathbb{C}^{F} \rightarrow\left(\mathbb{R}^{F}\right)^{*} \simeq \mathbb{R}^{F}$ given by

$$
\mu(z)_{f}=\left|z_{f}\right|^{2} / 2-\lambda_{f}=\left(x_{f}^{2}+y_{f}^{2}\right) / 2-\lambda_{f}, \quad f \in F .
$$

Here $z_{f}=x_{f}+\mathrm{i} y_{f}$, with $x_{f}, y_{f} \in \mathbb{R}$. Furthermore we work with the symplectic form

$$
\omega:=(\mathrm{i} / 4 \pi) \sum_{f \in F} \mathrm{~d} z_{f} \wedge \mathrm{d} \bar{z}_{f}=(1 / 2 \pi) \sum_{f \in F} \mathrm{~d} x_{f} \wedge \mathrm{d} y_{f} .
$$

The factor $1 / 2 \pi$ is introduced in order to avoid an integral lattice $(2 \pi \mathbb{Z})^{F}$ instead of our $\mathbb{Z}^{F}$.

Hence $N$ acts on $\mathbb{C}^{F}$ Hamiltonianly and the corresponding momentum mapping is $\mu_{N}:=\iota_{\mathfrak{n}}^{*} \circ \mu: \mathbb{C}^{F} \rightarrow \mathfrak{n}^{*}$, where $\iota_{\mathfrak{n}}: \mathfrak{n} \rightarrow \mathbb{R}^{F}$ denotes the identity viewed as a linear mapping from $\mathfrak{n} \subset \mathbb{R}^{F}$ to $\mathbb{R}^{F}$, and its transposed $\iota_{\mathfrak{n}}^{*}:\left(\mathbb{R}^{F}\right)^{*} \rightarrow \mathfrak{n}^{*}$ is the map which assigns to each linear form on $\mathbb{R}^{F}$ its restriction to $\mathfrak{n}$. 
Step 4 (The symplectic toric manifold $\left(M_{\Delta}, \omega_{\Delta}, T, \mu_{\Delta}\right)$ ). - It follows from Guillemin [34, Theorems 1.6 and 1.4] that 0 is a regular value of $\mu_{N}$, hence the zero level set $Z$ of $\mu_{N}$ is a smooth submanifold of $\mathbb{C}^{F}$, and that the action of $N$ on $Z$ is proper and free. The $N$-orbit space $M_{\Delta}:=Z / N$ is a smooth $2 n$-dimensional manifold such that the projection $p: Z \rightarrow M_{\Delta}$ exhibits $Z$ as a principal $N$-bundle over $M_{\Delta}$. Moreover, there is a unique symplectic form $\omega_{\Delta}$ on $M_{\Delta}$ such that $p^{*} \omega_{\Delta}=\iota_{Z}{ }^{*} \omega$, where $\iota_{Z}$ is the identity viewed as a smooth mapping from $Z$ to $\mathbb{C}^{F}$.

On $\left(M_{\Delta}, \omega_{\Delta}\right)$, the torus $\left(\mathbb{R}^{F} / \mathbb{Z}^{F}\right) / N \simeq T$ acts effectively and Hamiltonianly, with momentum mapping $\mu_{\Delta}: M_{\Delta} \rightarrow \mathfrak{t}^{*}$ determined by $\pi^{*} \circ \mu_{\Delta} \circ p=\left.\mu\right|_{Z}$, and ${ }^{(1)} \mu_{\Delta}\left(M_{\Delta}\right)=\Delta$. In other words, $\left(M_{\Delta}, \omega_{\Delta}, T, \mu_{\Delta}\right)$ is a symplectic toric manifold with momentum map image equal to $\Delta$.

Theorem 2.3 (Delzant's Theorem). - Any abstract symplectic toric manifold $(M, \omega, T, \mu)$ with momentum polytope $\Delta \subset \mathfrak{t}^{*}$ is isomorphic to $\left(M_{\Delta}, \omega_{\Delta}, T, \mu_{\Delta}\right)$. Moreover, two symplectic toric manifolds $\left(M_{\Delta}, \omega_{\Delta}, T, \mu_{\Delta}\right)$ and $\left(M_{\Delta^{\prime}}, \omega_{\Delta^{\prime}}, T, \mu_{\Delta^{\prime}}\right)$ are isomorphic if and only if $\Delta=\Delta^{\prime}$.

Since the action of $\mathbb{R}^{F} / \mathbb{Z}^{F}$ preserves the complex structure of $\mathbb{C}^{F}, M_{\Delta}$ inherits by reduction a complex structure compatible with $\omega_{\Delta}$ and invariant by the action of $T$ (cf. [37, Theorem 3.5]). So $M_{\Delta}$ is a Kähler manifold.

\section{Prequantization}

Let us recall the basic facts we need on connections of Hermitian line bundles. A good reference for this material are Duistermaat's notes [25]. See also [47, Sections 8-14] and [49].

Let $M$ be a manifold. Consider a Hermitian line bundle $\mathcal{L} \rightarrow M$, that is a complex line bundle endowed with a Hermitian metric. Let $\mathrm{C}^{\infty}(M, \mathscr{L})$ be the space of smooth sections of $\mathscr{L}$ and $\Omega^{1}(M, \mathcal{L})$ be the space of $\mathscr{L}$-valued 1 -forms. A connection of $\mathscr{L}$ is a linear operator

$$
\nabla: \mathrm{C}^{\infty}(M, \mathscr{L}) \rightarrow \Omega^{1}(M, \mathscr{L})
$$

satisfying the Leibniz rule

$$
\nabla(f s)=\mathrm{d} f \otimes s+f \nabla s, \quad \forall f \in \mathrm{C}^{\infty}(M), s \in \mathrm{C}^{\infty}(M, \mathscr{L}) .
$$

For any vector field $X$ of $M$, the covariant derivative of a section $s$ of $\mathcal{L}$ with respect to $X$ is $\nabla_{X} s=\nabla s(X)$. The curvature of the connection is the unique 2-form $R$ on $M$ satisfying for any vector fields $X, Y$ of $M$

$$
\left[\nabla_{X}, \nabla_{Y}\right]-\nabla_{[X, Y]}=R(X, Y) .
$$

The connection is compatible with the Hermitian structure if for any sections $s, t$ of $\mathscr{L}$,

$$
\mathrm{d}(s, t)=(\nabla s, t)+(s, \nabla t),
$$

where $(\cdot, \cdot)$ denotes the Hermitian scalar product. In that case, $R=\frac{1}{\mathrm{i}} \omega$ where $\omega$ is real valued. In this paper, we always assume the connections are compatible with the metric.

(1) See Guillemin [34, Theorem 1.7].

4 e SÉRIE - TOME $46-2013$ - No 5 
Assume that $\frac{1}{\mathrm{i}} \omega$ is the curvature of a Hermitian line bundle connection. Then the cohomology class of $\omega / 2 \pi$ is integral, in the sense that it belongs to the image of the natural homomorphism $\mathrm{H}^{2}(M, \mathbb{Z}) \rightarrow \mathrm{H}^{2}(M, \mathbb{R})$. Conversely, given any $\omega \in \Omega^{2}(M, \mathbb{R})$ such that $[\omega] / 2 \pi$ is integral, there exists a Hermitian line bundle $\mathcal{L}$ over $M$ with a connection $\nabla$ of curvature $\frac{1}{\mathrm{i}} \omega$. Furthermore, $\mathscr{L}$ and $\nabla$ are unique up to isomorphism. For a proof of these results, we refer the reader to [25, Theorem 10.1] or [26, Section 15.3].

Let $(M, \omega)$ be a symplectic manifold endowed with a prequantum bundle, that is a Hermitian line bundle $\mathcal{L} \rightarrow M$ with a connection of curvature $\frac{1}{\mathrm{i}} \omega$; such a symplectic manifold is called prequantizable. A prequantum bundle automorphism is a vector bundle automorphism of $\mathcal{L}$ preserving the metric and the connection. Let $G$ be a Lie group acting on $\mathscr{L}$ by prequantum bundle automorphisms. This action lifts an action of $G$ on $M$. One proves that the latter action is Hamiltonian and has a natural momentum map $\mu$ determined by the following condition: the induced action of the Lie algebra $\mathfrak{g}$ on $\mathrm{C}^{\infty}(M, \mathscr{L})$ is given by the Kostant-Souriau operators

$$
f \rightarrow \nabla_{X^{\sharp}} f+\mathrm{i}\langle\mu, X\rangle f, \quad X \in \mathfrak{g},
$$

where we denote by $X^{\sharp}$ the infinitesimal action of $X$ on $M$ and by $\nabla$ the covariant derivative of the prequantum bundle, cf. [26, Proposition 15.2]. If $G$ and $M$ are connected, the action on $\mathcal{L}$ is conversely determined by the action on $M$ and the momentum map $\mu$. However, not all momentum maps generating a given action can be obtained in this way. Actually these momentum maps correspond to the Lie algebra representations on the prequantum bundle, through Equation (3).

Assume now that $M$ is connected and $T$ is a torus acting on $M$ in a Hamiltonian way. The momentum map of this action is unique up to a translation by a vector of $t^{*}$. By [26, Proposition 15.4], this action can be lifted to $\mathcal{L}$ in such a way that each element acts as a prequantum bundle automorphism. This lift is not unique, actually the momentum map corresponding to a lift is unique up to a translation by a vector of $2 \pi \mathfrak{t}_{\mathbb{Z}}^{*}$. For a symplectic toric manifold, we can explicit everything in terms of the momentum polytope as follows.
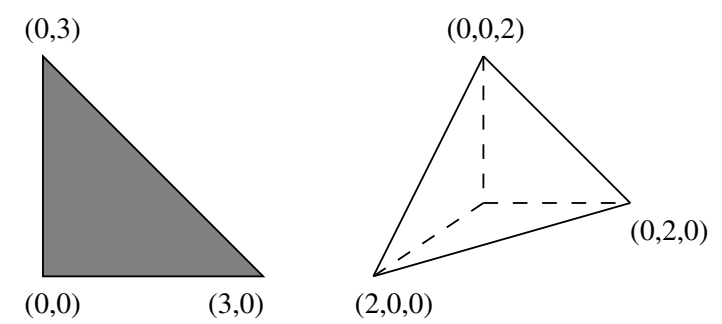

FIGURE 4. Delzant polytopes corresponding to the complex projective spaces $\mathbb{C P}^{2}$ and $\mathbb{C P}^{3}$ equipped with scalar multiples of the Fubini-Study symplectic form.

Proposition 3.1. - (a) A symplectic toric manifold $(M, \omega, T, \mu)$ with momentum polytope $\Delta \subset \mathfrak{t}^{*}$ admits a prequantum bundle $\mathcal{L}$ if and only if the edges of $\Delta$ belong to $2 \pi \mathfrak{t}_{\mathbb{Z}}^{*}$.

(b) When it exists, the prequantum bundle is unique up to isomorphism. 
(c) The momentum maps corresponding to the possible lifts (via (3)) are the ones such that the vertices of the associated polytope belong to $2 \pi \mathfrak{t}_{\mathbb{Z}}^{*}$.

Proof. - Let $(M, \omega)$ be a symplectic toric manifold with momentum map $\mu$. Let $e$ be a closed edge of $\Delta=\mu(M)$. Then $\mu^{-1}(e)$ is a symplectic 2-sphere embedded in $M$ with volume the length of $e$. Here the length of $e$ is the largest positive real $\ell$ such that $e / \ell \in \mathfrak{t}_{\mathbb{Z}}^{*}$. A proof of this fact may be found in [42, Lemma 2.10].

Now if $(M, \omega)$ is prequantizable, then $[\omega] / 2 \pi$ is integral. So necessarily the length of any edge is an integral multiple of $2 \pi$. Since $\mathrm{H}_{2}(M)$ is generated by the homology classes of the spheres corresponding to the various edges ([19, Proposition 10.6]), the condition is also sufficient. The uniqueness of the prequantum bundle follows from the fact that any symplectic toric manifold is simply connected (see [19, Th. 9.1]).

Assume now that $(M, \omega)$ has a prequantum bundle $\mathcal{L}$. We know that we can lift the action to $\mathcal{L}$. Then for any fixed point $p \in M$, the action preserves the fiber $\mathscr{L}_{p}$. Because of Formula (3), for any $\xi \in \mathfrak{t}, \exp (\xi)$ acts in $\mathcal{L}_{p}$ by multiplication by $\exp (\mathrm{i}\langle\mu(p), \xi\rangle)$. This implies that $\mu(p) \in 2 \pi \mathfrak{t}_{\mathbb{Z}}^{*}$. Since the fixed points of a toric manifold are the preimages of the vertices of the polytope, this shows that the vertices belong to $2 \pi \mathfrak{t}_{\mathbb{Z}}^{*}$.

In the sequel, we will use the following explicit construction of the prequantum bundle for the Delzant model. We use the same notations as in Section 2. Assume that the vertices of $\Delta$ belong to $2 \pi \mathfrak{t}_{\mathbb{Z}}^{*}$. Consider the prequantum bundle of $\mathbb{C}^{F}$ given by $\mathscr{L}_{F}:=\mathbb{C}^{F} \times \mathbb{C}$ with connection

$$
\nabla=d+\frac{1}{4 \pi \mathrm{i}} \sum_{f \in F}\left(x_{f} \mathrm{~d} y_{f}-y_{f} \mathrm{~d} x_{f}\right)=d+\frac{1}{8 \pi} \sum_{f \in F}\left(z_{f} \mathrm{~d} \bar{z}_{f}-\bar{z}_{f} \mathrm{~d} z_{f}\right) .
$$

Since the vertices of $\Delta$ belong to $2 \pi \mathfrak{t}_{Z}^{*}$, the $\lambda_{f}$ 's defining the faces of $\Delta$ are integral multiple of $2 \pi$. So we can lift the action of $\mathbb{R}^{F} / \mathbb{Z}^{F}$ on $\mathbb{C}^{F}$ to $\mathscr{L}_{F}$ by

$$
t \cdot(z, u)=\left(t \cdot z, u \mathrm{e}^{\mathrm{i} \sum_{f \in F} t_{f} \lambda_{f}}\right) .
$$

With a straightforward computation, one checks that this action preserves the prequantum bundle structure and that its associated momentum map is $\mu$ defined in (1).

Recall that the Delzant manifold $\left(M_{\Delta}, \omega_{\Delta}\right)$ is the quotient of $Z=\mu_{N}^{-1}(0)$ by the action of subtorus $N$ of $\mathbb{R}^{F} / \mathbb{Z}^{F}$. Then quotienting by $N$ the restriction of $\mathscr{L}_{F}$ to $Z$, we obtain a prequantum bundle $\mathscr{L}_{\Delta}$ over $M_{\Delta}$ (cf. [37, Theorem 3.2]). Furthermore the group $T=\left(\mathbb{R}^{F} / \mathbb{Z}^{F}\right) / N$ acts on $\mathscr{L}_{\Delta}$. This action preserves the prequantum bundle structure, its associated momentum is the application $\mu_{\Delta}$ defined in Theorem 2.3.

REMARK 3.2. - The construction of the prequantum bundle in the Delzant model gives an alternative proof of the fact that if the vertices of its momentum polytope $\Delta$ belong to $2 \pi \mathfrak{t}_{\mathbb{Z}}^{*}$, a symplectic toric manifold is prequantizable. Moreover, the converse statement can be deduced directly from the last paragraph of the proof of Proposition 3.1: indeed, the polytope $(2 \pi)^{-1} \Delta$ can be translated to a polytope with integral vertices if and only if its edges have integral lengths. 


\section{Quantum model}

In this section we introduce a quantum model for quantum toric system and compute its spectrum.

Consider a Delzant polytope $\Delta \subset \mathfrak{t}^{*}$ with vertices in $2 \pi \mathfrak{t}_{\mathbb{Z}}^{*}$. Then as explained in Section 3 , the Delzant manifold $\left(M_{\Delta}, \omega_{\Delta}, T, \mu_{\Delta}\right)$ admits a prequantum bundle $\mathscr{L}_{\Delta}$ unique up to isomorphisms. This line bundle has a unique holomorphic structure compatible with the complex structure of $M_{\Delta}$ provided by reduction and with the connection. So for any positive integer $k$, we can define the quantum space

$$
\mathcal{H}_{k}^{\Delta}:=\mathrm{H}^{0}\left(M_{\Delta}, \mathscr{L}_{\Delta}^{k}\right)
$$

which consists of the holomorphic sections of $\mathcal{L}_{\Delta}^{k}$.

For any $X \in \mathfrak{t}$, consider the rescaled Kostant-Souriau operator

$$
T_{X, k}:=\left\langle\mu_{\Delta}, X\right\rangle+\frac{1}{\mathrm{i} k} \nabla_{X^{\sharp}}: \mathcal{H}_{k}^{\Delta} \rightarrow \mathcal{H}_{k}^{\Delta} .
$$

This operator is well-defined because the complex structure is invariant by the action of $T$ on $M_{\Delta}$. The rescaling has the effect that the $T_{X, k}$ 's are self-adjoint and that their joint spectrum is the intersection of the polytope $\Delta$ and a rescaled lattice. The precise result is the following.

Theorem 4.1. - There is an orthogonal decomposition of the quantum space $\mathcal{H}_{k}^{\Delta}$ into a direct sum of lines:

such that, for any $X \in \mathfrak{t}$,

$$
\mathcal{H}_{k}^{\Delta}=\bigoplus_{\ell \in\left(\frac{2 \pi}{k} t_{\mathbb{Z}}^{*}\right) \cap \Delta} D_{\ell}^{k}
$$

$$
T_{X, k} \Psi=\ell(X) \Psi, \quad \text { for all } \Psi \in D_{\ell}^{k} .
$$

Proof. - The proof uses the Delzant construction recalled in Section 2 at the quantum level. Consider the same prequantum bundle $\mathscr{L}_{F} \rightarrow \mathbb{C}^{F}$ as in the proof of Proposition 3.1. The associated quantum space $\mathscr{B}_{k}$ is the space of holomorphic sections $\psi$ of $\mathcal{L}_{F}^{k}$ such that

$$
\int_{\mathbb{C}^{F}}|\psi|^{2}(z) \nu(z)<\infty
$$

where $\nu$ is the Liouville measure. Here holomorphic means that the covariant derivative with respect to antiholomorphic vectors vanishes. Such a holomorphic section can be written

$$
\psi=\mathrm{e}^{-k|z|^{2} / 8 \pi} f
$$

where $f$ is a plain holomorphic function on $\mathbb{C}^{F}$. Here $|z|^{2}=\sum_{f}\left|z_{f}\right|^{2}$. So $\mathscr{B}_{k}$ can be identified with the usual Bargmann space, that is the space of holomorphic functions on a $\mathbb{C}^{p}$ whose square is integrable with respect to a given gaussian weight. An orthogonal basis of $\mathscr{B}_{k}$ is given by the family

$$
\psi_{\alpha}=\mathrm{e}^{-\frac{k}{8 \pi}|z|^{2}} z^{\alpha}, \quad \alpha \in \mathbb{N}^{F}
$$

Consider the Kostant-Souriau operator associated to momentum $\mu$ given in (1)

$$
S_{X, k}=\langle\mu, X\rangle+\frac{1}{\mathrm{i} k} \nabla_{X^{\sharp}}
$$


If $X=e_{f}$ a straightforward computation shows that

$$
S_{X, k}\left(\mathrm{e}^{-\frac{k}{8 \pi}|z|^{2}} g(z)\right)=\mathrm{e}^{-\frac{k}{8 \pi}|z|^{2}}\left(\frac{2 \pi}{k} z_{f} \partial_{z_{f}} g-\lambda_{f} g\right) .
$$

We deduce that for any $X \in \mathbb{R}^{F}$ and $\alpha \in \mathbb{N}^{F}$,

$$
S_{X, k}\left(\psi_{\alpha}\right)=\left\langle X, \frac{2 \pi}{k} \alpha-\lambda\right\rangle \psi_{\alpha}
$$

Recall that the Delzant space was defined as the symplectic quotient of $\mathbb{C}^{F}$ by the subtorus $N$ of $\mathbb{R}^{F} / \mathbb{Z}^{F}$. The corresponding space at the quantum level is

$$
\mathscr{B}_{k}^{\mathfrak{n}}:=\left\{\psi \in \mathscr{B}_{k} \quad \mid \quad S_{X, k} \psi=0 \quad \text { for all } X \in \mathfrak{n}\right\} .
$$

We call it the reduced quantum space. We deduce from Equation (5) that a basis of $\mathcal{B}_{k}^{\mathfrak{n}}$ consists of the $\Psi_{\alpha}$ 's such that $\alpha \in \mathbb{N}^{F}$ satisfies

$$
\left\langle X, \frac{2 \pi}{k} \alpha-\lambda\right\rangle=0
$$

for all $X \in \mathfrak{n}$. Equivalently, $\frac{2 \pi}{k} \alpha$ runs over $\left(\frac{2 \pi}{k} \mathbb{N}^{F}\right) \cap\left(\lambda+\operatorname{ker}\left(\iota_{\mathfrak{n}}^{*}\right)\right)$. This set is in bijection with $\left(\frac{2 \pi}{k} \mathfrak{t}_{\mathbb{Z}}^{*}\right) \cap \Delta$.

Lemma 4.2. - The map $\pi^{*}+\lambda$ from $\mathfrak{t}^{*}$ to $\left(\mathbb{R}^{F}\right)^{*}$ restricts to a bijection

$$
\left(\frac{2 \pi}{k} \mathfrak{t}_{\mathbb{Z}}^{*}\right) \cap \Delta \longrightarrow\left(\frac{2 \pi}{k} \mathbb{N}^{F}\right) \cap\left(\lambda+\operatorname{ker}\left(\iota_{\mathfrak{n}}^{*}\right)\right)
$$

Furthermore, for any $X \in \mathbb{R}^{F}$ and $\ell \in \frac{2 \pi}{k} \mathfrak{t}_{\mathbb{Z}}^{*} \cap \Delta$ we have that

$$
S_{X, k}\left(\psi_{\alpha}\right)=\langle\ell, \pi(X)\rangle \psi_{\alpha}, \quad \text { if } \quad \frac{2 \pi}{k} \alpha=\pi^{*}(\ell)+\lambda .
$$

Proof of Lemma 4.2. - We know that $\pi^{*}$ is injective with image $\operatorname{ker}\left(\iota_{\mathfrak{n}}^{*}\right)$. Using the Delzant condition on a vertex (Step 1 in Section 2), one sees that $\pi^{*}$ restricts to a bijection from $\mathfrak{t}_{\mathbb{Z}}^{*}$ to $\left(\mathbb{Z}^{F}\right)^{*} \cap \operatorname{ker}\left(\iota_{\mathfrak{n}}^{*}\right)$. By the prequantization condition, $\lambda \in 2 \pi\left(\mathbb{Z}^{F}\right)^{*} \subset \frac{2 \pi}{k}\left(\mathbb{Z}^{F}\right)^{*}$. So $\pi^{*}+\lambda$ restricts to a bijection from $\frac{2 \pi}{k} \mathfrak{t}_{\mathbb{Z}}^{*}$ to $\left(\frac{2 \pi}{k} \mathbb{Z}^{F}\right) \cap\left(\lambda+\operatorname{ker}\left(\iota_{\mathfrak{n}}^{*}\right)\right)$. Furthermore, the proof to show that $\mu_{\Delta}\left(M_{\Delta}\right)=\Delta$ implies that

$$
\pi^{*}(\Delta)+\lambda=\mathbb{R}_{\geq 0}^{F} \cap\left(\lambda+\operatorname{ker}\left(\iota_{\mathfrak{n}}^{*}\right)\right)
$$

This implies the first part of the lemma. The second assertion follows from (5).

The end of the proof of Theorem 4.1 is an application of the "quantization commutes with reduction" theorem of Guillemin-Sternberg. Lifting the action of $\mathbb{T}^{F}=\mathbb{R}^{F} / \mathbb{Z}^{F}$ to $\mathscr{L}_{F}$ as in the proof of Proposition 3.1, we get an action of $\mathbb{T}^{F}$ on $\mathscr{B}_{k}$. The reduced quantum space is the subspace of $\mathscr{B}_{k}$ of $N$-invariant vectors, in other words $\mathscr{B}_{k}^{\mathfrak{n}}=\left(\mathscr{B}_{k}\right)^{N}$. Now by GuilleminSternberg theorem ([37]), we have an isomorphism

$$
\Phi_{k}: \mathscr{B}_{k}^{\mathfrak{n}} \rightarrow \mathscr{H}_{k}^{\Delta}
$$

The proof in [37] given in the compact case extends to our setting by [10] and [34]. Furthermore, under the isomorphism $\Phi_{k}$, the action of the torus $T$ on $\mathcal{H}_{k}^{\Delta}$ corresponds to the action 
of $\mathbb{T}^{F} / N$ on $\mathscr{B}_{k}^{\mathfrak{n}}$. Then, passing to the level of Lie algebras, we get the following relation between the Kostant-Souriau operators:

$$
\Phi_{k}\left(S_{X, k} \Psi\right)=T_{\pi(X), k} \Phi_{k}(\Psi), \quad \text { for all } \Psi \in \mathscr{B}_{k}^{\mathfrak{n}} .
$$

This concludes the proof of Theorem 4.1.

From the previous theorem, we deduce the following quantum normal form. Consider a Delzant polytope $\Delta$ in the Lie algebra $\mathbb{R}^{n}$ of $\mathbb{R}^{n} / \mathbb{Z}^{n}$. Assume $\left(M_{\Delta}, \omega_{\Delta}\right)$ has a prequantum bundle $\mathscr{L}_{\Delta}$ and define the associated quantum spaces

$$
\mathscr{H}_{k}^{\Delta}=\mathrm{H}^{0}\left(M_{\Delta}, \mathcal{L}_{\Delta}^{k}\right)
$$

Starting from the canonical basis $\left(e_{i}\right)$ of $\mathbb{R}^{n}$, we get $n$ operators

$$
T_{i, k}^{\Delta}:=T_{e_{i}, k}: \mathcal{H}_{k}^{\Delta} \rightarrow \mathcal{H}_{k}^{\Delta}, \quad k \in \mathbb{Z}_{>0}, 1 \leqslant i \leqslant n,
$$

defined by Kostant-Souriau Formula (4).

Corollary 4.3. - For any $k, T_{1, k}^{\Delta}, \ldots, T_{n, k}^{\Delta}$ are mutually commuting operators with simple joint eigenspaces. Their joint spectrum is $\left(v+\frac{2 \pi}{k} \mathbb{Z}^{n}\right) \cap \Delta$ where $v$ is any vertex of $\Delta$.

Proof. - Apply Theorem 4.1 to the polytope $\Delta-v$, whose vertices are integral.

\section{Global quantum normal form}

\section{Toeplitz operators}

We briefly review Toeplitz operators. Let $(M, \omega)$ be a compact connected symplectic manifold with a prequantum line bundle $\mathcal{L}$. Assume that $M$ is endowed with a complex structure $j$ compatible with $\omega$, so that $M$ is Kähler and $\mathscr{L}$ is holomorphic. Here the holomorphic structure of the prequantum bundle is the unique one compatible with the connection. Recall that for a positive integer $k, \mathcal{H}_{k}:=\mathrm{H}^{0}\left(M, \mathscr{L}^{k}\right)$ is the space of holomorphic sections of $\mathscr{L}^{k}$.

Since $M$ is compact, $\mathscr{H}_{k}$ is a closed finite dimensional subspace of the Hilbert space $\mathrm{L}^{2}\left(M, \mathcal{L}^{k}\right)$. Here the scalar product is defined by integrating the Hermitian pointwise scalar product of sections against the Liouville measure of $M$. Denote by $\Pi_{k}$ the orthogonal projector of $\mathrm{L}^{2}\left(M, \mathcal{L}^{k}\right)$ onto $\mathscr{H}_{k}$.

A Toeplitz operator is any sequence $\left(T_{k}: \mathcal{H}_{k} \rightarrow \mathcal{H}_{k}\right)_{k \in \mathbb{N}^{*}}$ of operators of the form

$$
\left(T_{k}=\Pi_{k} f(\cdot, k)+R_{k}\right)_{k \in \mathbb{N}^{*}}
$$

where $f(\cdot, k)$, viewed as a multiplication operator, is a sequence in $\mathrm{C}^{\infty}(M)$ with an asymptotic expansion $f_{0}+k^{-1} f_{1}+\cdots$ for the $\mathrm{C}^{\infty}$ topology, and the norm of $R_{k}$ is $\theta\left(k^{-\infty}\right)$.

We denote by $\mathscr{T}(M, \mathscr{L}, j)$ the set of Toeplitz operators. The following result corresponds to [10, Theorem 1.2]. 
Theorem 5.1. - The set $\mathscr{T}=\mathscr{T}(M, \mathcal{L}, j)$ is a semiclassical algebra associated to $(M, \omega)$ in the following sense. The set $\mathscr{T}$ is closed under the formation of product. So it is a star algebra, the identity is $\left(\Pi_{k}\right)_{k \in \mathbb{N}^{*}}$. The symbol map $\sigma_{\text {cont }}: \mathcal{T} \rightarrow \mathrm{C}^{\infty}(M)[[\hbar]]$, sending $T_{k}$ into the formal series $f_{0}+\hbar f_{1}+\cdots$ where the functions $f_{i}$ are the coefficients of the asymptotic expansion of the multiplier $f(\cdot, k)$, is well defined. It is onto and its kernel is the ideal consisting of $\Theta\left(k^{-\infty}\right)$ Toeplitz operators. More precisely for any integer $\ell$,

$$
\left\|T_{k}\right\|=\Theta\left(k^{-\ell}\right) \text { if and only if } \sigma_{\mathrm{cont}}\left(T_{k}\right)=\Theta\left(\hbar^{\ell}\right) .
$$

Furthermore, the induced product $*_{\text {cont }}$ on $\mathrm{C}^{\infty}(M)[[\hbar]]$ is a star-product.

We call the formal series

$$
\sigma_{\text {cont }}\left(T_{k}\right)=f_{0}+\hbar f_{1}+\cdots
$$

the contravariant symbol of $\left(T_{k}\right)_{k \in \mathbb{N}^{*}}$. The first coefficient $f_{0}$ is the principal symbol of $\left(T_{k}\right)_{k \in \mathbb{N}^{*}}$. The subprincipal symbol of $\left(T_{k}\right)_{\in \mathbb{N}^{*}}$ is the function

$$
g_{1}=f_{1}+\frac{1}{2} \Delta f_{0}
$$

where $\Delta$ is the holomorphic Laplacian of $M$.

Consider two Toeplitz operators with principal and subprincipal symbols $g_{0}, g_{1}$ and $g_{0}^{\prime}, g_{1}^{\prime}$ respectively. Then the principal and subprincipal symbol of their composition is

$$
g_{0}^{\prime \prime}+\hbar g_{1}^{\prime \prime}=\left(g_{0}+\hbar g_{1}\right)\left(g_{0}^{\prime}+\hbar g_{1}^{\prime}\right)+\frac{\hbar}{2 i}\left\{g_{0}, g_{0}^{\prime}\right\}+O\left(\hbar^{2}\right)
$$

where $\{\cdot, \cdot\}$ is the Poisson bracket of $(M, \omega)$ (cf. Theorem 1.4 of [9]).

The Kostant-Souriau operators considered in Section 4 are Toeplitz operators. More generally, let $f$ be a function of $M$ with Hamiltonian vector field $X$. Applying the Tuynman's trick ([68]), one proves that the sequence

$$
T_{k}:=\Pi_{k}\left(f+\frac{1}{\mathrm{i} k} \nabla_{X}\right), \quad k \in \mathbb{N}^{*},
$$

is a Toeplitz operator with principal symbol $f$ and subprincipal symbol $-\frac{1}{2} \Delta f$.

\section{Normal Form}

Recall that for each Delzant polytope $\Delta \subset \mathbb{R}^{n}$, we introduced in Section 2 a symplectic toric manifold $\left(M_{\Delta}, \omega_{\Delta}, \mathbb{R}^{n} / \mathbb{Z}^{n}, \mu_{\Delta}\right)$, a complex structure $j_{\Delta}$ on $M_{\Delta}$ compatible with $\omega_{\Delta}$. Assume that $\Delta+c$ has integral vertices for some $c \in \mathbb{R}^{n}$, so that $\left(M_{\Delta}, \omega_{\Delta}\right)$ has a prequantum bundle $\mathscr{L}_{\Delta}$ (unique up to isomorphism). We defined in Section 4 for any positive $k$, commuting operators $T_{1, k}^{\Delta}, \ldots, T_{n, k}^{\Delta}$ acting on the Hilbert spaces $\mathcal{H}_{k}^{\Delta}=\mathrm{H}^{0}\left(M_{\Delta}, \mathscr{L}_{\Delta}^{k}\right), k \in \mathbb{Z}_{>0}$, and described explicitly their spectrum in Corollary 4.3.

THEOREM 5.2 (Global normal form for a quantum toric system)

Let $\left(M, \omega, \mathbb{R}^{n} / \mathbb{Z}^{n}, \mu\right)$ be a symplectic toric manifold equipped with a prequantum bundle $\mathcal{L}$ and a compatible complex structure $j$. Denote by $\Delta$ the momentum polytope $\mu(M) \subset \mathbb{R}^{n}$. Let $T_{1}, \ldots, T_{n}$ be commuting Toeplitz operators of $\mathscr{T}(M, \mathcal{L}, j)$ whose principal symbols are the components of $\mu$.

4 e SÉRIE - TOME $46-2013$ - No 5 
Then there exists $k_{0}>0$, there exists a sequence $(g(\cdot ; k))_{k \geqslant k_{0}}$ of smooth maps $\mathbb{R}^{n} \rightarrow \mathbb{R}^{n}$, and there exists an operator $U=\left(U_{k}: \mathcal{H}_{k} \rightarrow \mathcal{H}_{k}^{\Delta}\right)_{k \geqslant k_{0}}$ with $U_{k}$ invertible for any $k$, such that

$$
U_{k}\left(T_{1, k}, \ldots, T_{n, k}\right) U_{k}^{-1}=g\left(T_{1, k}^{\Delta}, \ldots, T_{n, k}^{\Delta} ; k\right)+\Theta\left(k^{-\infty}\right) .
$$

Moreover, $g$ admits an asymptotic expansion in the $\mathrm{C}^{\infty}$ topology of the form

$$
g(\cdot ; k)=\mathrm{Id}+k^{-1} g_{1}+k^{-2} g_{2}+\cdots .
$$

If the operators $T_{j}$ are self-adjoint (i.e., for any $k, T_{j, k}$ is a self-adjoint operator), then $U_{k}$ may be chosen such that $U_{k} U_{k}^{*}=\operatorname{Id}_{\mathcal{H}_{k}^{\Delta}}$.

Remark 5.3. - For small $k$, the dimensions of $\mathscr{H}_{k}$ and $\mathcal{H}_{k}^{\Delta}$ might be different. Theorem 5.2 does not give information about small values of $k$.

The proof of Theorem 5.2 will require the following technical lemma, which is a global version of a result of Eliasson [30, Corollary page 14]. Recall that in the case where $E$ is a closed half-space, $h \in \mathrm{C}^{\infty}\left(\mathbb{R}_{x}^{n} \times E\right)$ if and only if all the partial derivatives $\partial_{x}^{k} \partial_{e}^{\ell} h(x, e)$ for $(x, e) \in \mathbb{R}^{n} \times \stackrel{\circ}{E}$ have a limit at every point in $\mathbb{R}^{n} \times E$. (This is equivalent to saying that $g$ has a smooth extension in a neighborhood of any point.)

Lemma 5.4. - Let $E$ be a vector space or a closed half-space. Let $f \in \mathrm{C}^{\infty}\left(\mathbb{R}_{(x, \xi)}^{2} \times E\right)$, and let

$$
q(x, \xi, e)=x^{2}+\xi^{2} .
$$

Assume that $\{q, f\}=0$ (here the Poisson bracket refers to the symplectic variables $(x, \xi)$ ). Then there exists $g \in \mathrm{C}^{\infty}\left(\mathbb{R}_{\geqslant 0} \times E\right)$ such that

$$
f(x, \xi, e)=g(q(x, \xi, e), e) .
$$

Proof. - Set-theoretically, there is a unique such function $g$. We need to prove that $g$ is smooth. The Taylor expansion of $f$ in the $(x, \xi)$ variables has to commute with $q$. This implies that it has the form

$$
\sum_{k \geqslant 0} q^{k} a_{k}(e)
$$

where $a_{k} \in \mathrm{C}^{\infty}(E)$. Hence by the Taylor formula, for any integer $r \geqslant 0$, there is a polynomial $P_{r}$ in $q$ with coefficients in $\mathrm{C}^{\infty}(E)$, and a smooth function $\varphi \in \mathrm{C}^{\infty}\left(\mathbb{R}^{2} \times E\right)$ such that

$$
f=P_{r}(q, e)+q^{r} \varphi(x, \xi, e)
$$

Thus we get

$$
g(t, e)=P_{r}(t, e)+t^{r} \varphi(\sqrt{t}, 0, e) .
$$

When $t>0$, we simply compute the partial derivatives $\partial_{t}^{k} \partial_{e}^{\ell} g(t, e)$. They have a limit as $(t, e) \rightarrow(0, e)$ as long as $k \leqslant r$. Thus $g \in \mathrm{C}^{r}\left(\mathbb{R}_{\geqslant 0} \times E\right)$, which proves the lemma.

Proof of Theorem 5.2. - We divide the proof into several steps. 
Step 1. - By Theorem 2.3, there exists a symplectomorphism $\varphi: M \rightarrow M_{\Delta}$ such that $\mu=\varphi^{*} \mu_{\Delta}$. Since $M_{\Delta}$ is simply connected, the prequantum bundle $\mathscr{L}_{\Delta}$ is unique up to isomorphism. Hence $\varphi$ can be lifted to a prequantum bundle isomorphism $\mathcal{L} \rightarrow \mathscr{L}_{\Delta}$. So $\varphi$ can be quantized as an operator $U_{k}: \mathcal{H}_{k} \rightarrow \mathcal{H}_{k}^{\Delta}$ such that $U_{k} U_{k}^{*}=I_{k}$ for large $k$ and such that for any Toeplitz operator $S=S_{k}$ with principal symbol $s, U_{k} S U_{k}^{*}$ is a Toeplitz operator whose principal symbol is $s \circ \varphi^{-1}$.

The operators $U_{k}$ that we use here have been introduced in [8, Chapter 4] and similar ones have been considered by [74]. They are analogues of Fourier integral operators [39, 27].

Replacing $T_{j}$ by $U T_{j} U^{*}$ we see that the problem is reduced to the case where $T_{1}, \ldots, T_{n}$ are commuting Toeplitz operators on $\left(M_{\Delta}, \mathscr{L}_{\Delta}\right)$, with joint principal symbol equal to $\mu_{\Delta}$.

Step 2. - We now prove the theorem by induction. Assume that, for some $N \in \mathbb{N}$, we have

$$
\left(T_{1}, \ldots, T_{n}\right)=g^{(N)}\left(T_{1}^{\Delta}, \ldots, T_{n}^{\Delta} ; k\right)+k^{-(N+1)} R_{N+1},
$$

where $R_{N+1}$ is a vector of $n$ Toeplitz operators and $g^{(N)}$ is polynomial in $k$ :

$$
g^{(N)}=\mathrm{Id}+k^{-1} g_{1}+\cdots+k^{-N} g_{N}
$$

and each $g_{j}: \mathbb{R}^{n} \rightarrow \mathbb{R}^{n}$ is a smooth map. For simplicity we write

$$
G_{N}:=g^{(N)}\left(T_{1}^{\Delta}, \ldots, T_{n}^{\Delta} ; k\right) .
$$

Notice that, for $N=0$, this is precisely the result of Step 1 . We wish to prove that there exists an invertible Toeplitz operator $U=\left(U_{k}\right)_{k \in \mathbb{N}}$ such that

$$
U\left(T_{1}, \ldots, T_{n}\right) U^{-1}=g^{(N)}\left(T^{\Delta} ; k\right)+k^{-(N+1)} h_{N+1}\left(T^{\Delta}\right)+k^{-(N+2)} R_{N+2} .
$$

The procedure is standard and we only indicate the key points. It turns out that the case $N=0$ is slightly different from the other cases $N>0$. When $N=0$, we plug (8) in the left-hand side of (9) and multiply on the right by $U$, and obtain

$$
\left[U, T^{\Delta}\right]+k^{-1} U R_{1}=k^{-1} G_{1} U \bmod k^{-2} \mathscr{T} .
$$

Since both sides of the equation are Toeplitz operators of order 1, the equation is equivalent to the equality of the principal symbols :

$$
\frac{1}{i}\left\{u, \mu_{\Delta}\right\}+u r_{1}=g_{1}\left(\mu_{\Delta}\right) u .
$$

Writing $u$ of the form $u=\mathrm{e}^{i a}$ we get the equation

$$
\left\{\mu_{\Delta}, a\right\}=r_{1}-g_{1}\left(\mu_{\Delta}\right) .
$$

For $N \geqslant 1$ we look for $U$ in the form $U_{k}=\mathrm{Id}+\mathrm{i} k^{-N} A_{N} \bmod k^{-(N+1)} \mathscr{T}$, where $A_{N}$ is a Toeplitz operator. The same calculation as before gives the equation

$$
\mathrm{i} k^{-N}\left[A_{N}, G_{N}\right]+k^{-(N+1)}\left(R_{N+1}-K_{N+1}\right)=0 \quad \bmod k^{-(N+2)} \mathscr{T} .
$$

(We use here $2 N+1 \geqslant N+2$ in order to eliminate higher order terms.) Since

$$
G_{N}=\left(T_{1}^{\Delta}, \ldots T_{n}^{\Delta}\right)+\Theta(1)
$$

the equation is equivalent to the following equation on the principal symbols :

$$
\left\{\mu_{\Delta}, a_{N}\right\}=r_{N+1}-h_{N+1}\left(\mu_{\Delta}\right) .
$$

$4^{\text {e }}$ SÉRIE - TOME $46-2013$ - No 5 
Step 3. - In order to complete the induction, we need to solve the following cohomological equation, where the unknown functions are $a$ and $g_{j}, 1 \leqslant j \leqslant n$ :

$$
\left\{\mu_{j}^{\Delta}, a\right\}=r_{j}-g_{j} \circ \mu_{\Delta}, \quad 1 \leqslant j \leqslant n .
$$

The proof follows Eliasson's local argument in [30, Lemma 8], where he uses a formula due to Moser. Here we show that this local argument also works globally.

For any smooth function $r$ on $M_{\Delta}$, we define

$$
M_{j} r=\int_{0}^{1} r \circ \varphi_{j}^{t} \mathrm{~d} t, \quad P_{j} r:=\int_{0}^{1} t r \circ \varphi_{j}^{t} \mathrm{~d} t .
$$

$M_{j}$ and $P_{j}$ are clearly linear operators sending $\mathrm{C}^{\infty}\left(M_{\Delta}\right)$ into itself. Notice that, since the flows $\varphi_{j}$ pairwise commute, the Fubini formula ensures that $M_{j}$ and $P_{k}$ commute for any $j, k$. The following Poisson bracket is easy to compute :

$$
\begin{aligned}
\left\{\mu_{j}^{\Delta}, P_{j} r\right\} & =\int_{0}^{1} t\left\{\mu_{j}^{\Delta}, r \circ \varphi_{j}^{t}\right\} \mathrm{d} t=\int_{0}^{1} t\left\{\mu_{j}^{\Delta} \circ \varphi_{j}^{t}, r \circ \varphi_{j}^{t}\right\} \mathrm{d} t=\int_{0}^{1} t\left\{\mu_{j}^{\Delta}, r\right\} \circ \varphi_{j}^{t} \mathrm{~d} t \\
& =\int_{0}^{1} t \frac{\mathrm{d}}{\mathrm{d} t}\left(r \circ \varphi_{j}^{t}\right) \mathrm{d} t=r-\int_{0}^{1} r \circ \varphi_{j}^{t}=r-M_{j} r .
\end{aligned}
$$

We shall need the following lemmas .

Lemma 5.5. - Let $r_{1}, \ldots, r_{n}$ be smooth functions on $M_{\Delta}$ such that for all $i, j$,

$$
\left\{\mu_{i}^{\Delta}, r_{j}\right\}=\left\{\mu_{j}^{\Delta}, r_{i}\right\}
$$

then for all $1 \leqslant i, j \leqslant n$, we have that $\left\{M_{j} r_{j}, \mu_{i}^{\Delta}\right\}=0$.

Lemma 5.6. - Let $f \in \mathrm{C}^{\infty}(M)$ such that for all $1 \leqslant i \leqslant n$ we have that $\left\{\mu_{i}^{\Delta}, f\right\}=0$. Then there exists $g \in \mathrm{C}^{\infty}\left(\mathbb{R}^{n}\right)$ such that $f=g \circ \mu_{\Delta}$.

Proof of Lemma 5.5. - We have that

$$
\begin{aligned}
\left\{\mu_{i}^{\Delta}, M_{j} r_{j}\right\} & =\int_{0}^{1}\left\{\mu_{i}^{\Delta}, r_{j} \circ \varphi_{j}^{t}\right\} \mathrm{d} t=\int_{0}^{1}\left\{\mu_{i}^{\Delta}, r_{j}\right\} \circ \varphi_{j}^{t} \mathrm{~d} t=\int_{0}^{1}\left\{\mu_{j}^{\Delta}, r_{i}\right\} \varphi_{j}^{t} \mathrm{~d} t \\
& =\int_{0}^{1} \frac{\mathrm{d}}{\mathrm{d} t}\left(r_{i} \circ \varphi_{j}^{t}\right) \mathrm{d} t=0,
\end{aligned}
$$

as desired.

Proof of Lemma 5.6. - Since $f$ is invariant by the action, set-theoretically, there exists a unique function $g$ such that $f=g \circ \mu_{\Delta}$. We want to prove that $g$ is smooth.

At a regular value of $\mu_{\Delta}$, this follows directly from the action-angle theorem. Let $c$ be a critical value of $\mu_{\Delta}$, and let $C$ be a small ball around $c$. Let $\left(z_{1}, \ldots, z_{k}, I_{1}, \theta_{1}, \ldots, I_{\ell}, \theta_{\ell}\right) \in$ $\mathbb{C}^{k} \times\left(\mathrm{T}^{*} S^{1}\right)^{\ell}$, with $k+\ell=n$ be Delzant coordinates on $\left(\mu_{\Delta}\right)^{-1}(C)$. Up to an affine transformation, we can assume

$$
\mu_{\Delta}=\left(\left|z_{1}\right|^{2} / 2, \ldots,\left|z_{k}\right|^{2} / 2, I_{1}, \ldots, I_{\ell}\right) .
$$

By assumption, $f$ does not depend on the $\theta_{j}$ coordinates, so there is a smooth function $g_{0}$ such that $f=g_{0}\left(z_{1}, \ldots, z_{k}, I_{1}, \ldots, I_{\ell}\right)$. 
We apply Lemma 5.4 to the function $g_{0}$ with $(x, \xi)=z_{1}$ and $E=\mathbb{C}^{k-1} \times \mathbb{R}^{\ell}$. Thus there is a smooth function $g_{1}$ such that

$$
f=g_{1}\left(\left|z_{1}\right|^{2}, z_{2}, \ldots, z_{k}, I_{1}, \ldots, I_{\ell}\right) .
$$

We may now apply the same lemma to

$$
f_{1}(z, e)=g\left(e_{1}, z, e_{2}, \ldots, e_{k}, e_{k+1}, \ldots, e_{n}\right)
$$

with $E=\mathbb{R}_{\geqslant 0} \times \mathbb{C}^{k-1} \times \mathbb{R}^{\ell}$ and get a smooth function $g_{2}$ such that

$$
f=g_{2}\left(\left|z_{1}\right|^{2},\left|z_{2}\right|^{2}, z_{3}, \ldots, z_{k}, I_{1}, \ldots, I_{\ell}\right) .
$$

We may repeat the argument and finally obtain a smooth function $g_{k}$ such that

$$
f=g_{k}\left(\left|z_{1}\right|^{2}, \ldots,\left|z_{k}\right|^{2}, I_{1}, \ldots, I_{\ell}\right) .
$$

This proves that $g$ is smooth in $C$. Thus $g$ is smooth on $\mu_{\Delta}\left(M_{\Delta}\right)$ (which means that there is a smooth extension of $g$ in $\mathbb{R}^{n}$ ).

We return now to the cohomological Equation (10). By Lemmas 5.5 and 5.6, there exist smooth functions $g_{j}$ on $\mathbb{R}^{n}$ such that $M_{j} r_{j}=g_{j} \circ \mu_{\Delta}$. Let

$$
a=P_{1} r_{1}+P_{2} M_{1} r_{2}+P_{3} M_{2} M_{1} r_{3}+\cdots+P_{n} M_{n-1} \cdots M_{1} r_{n} .
$$

Notice that for any function $h,\left\{\mu_{j}^{\Delta}, M_{j} h\right\}=0$. Hence, since the operators $M_{j}$ and $P_{k}$ commute, we get

$$
\left\{\mu_{1}^{\Delta}, a\right\}=\left\{\mu_{1}^{\Delta}, P_{1} r_{1}\right\}=r_{1}-M_{1} r_{1}=r_{1}-g_{1} \circ \mu_{\Delta},
$$

so $a$ solves the first equation of (10). Let

$$
\tilde{a}=a-P_{1} r_{1} .
$$

We have $\left\{\mu_{1}^{\Delta}, \tilde{a}\right\}=0$, and our system becomes

$$
\left\{\mu_{j}^{\Delta}, \tilde{a}\right\}=\tilde{r}_{j}-g_{j} \circ \mu_{\Delta}, \quad j=1, \ldots, n .
$$

with

$$
\begin{aligned}
\tilde{r}_{j} & :=r_{j}-\left\{\mu_{j}^{\Delta}, P_{1} r_{1}\right\}=r_{j}-\int_{0}^{1} t\left\{\mu_{j}^{\Delta}, r_{1} \circ \varphi_{1}^{t}\right\} \mathrm{d} t=r_{j}-\int_{0}^{1} t\left\{\mu_{1}^{\Delta}, r_{j}\right\} \circ \varphi_{1}^{t} \mathrm{~d} t \\
& =r_{j}-\int_{0}^{1} t \frac{\mathrm{d}}{\mathrm{d} t}\left(r_{j} \circ \varphi_{1}^{t}\right) \mathrm{d} t=M_{1} r_{j} .
\end{aligned}
$$

We notice that

$$
\tilde{a}=P_{2} \tilde{r}_{2}+P_{3} M_{2} \tilde{r}_{3}+\cdots+P_{n} M_{n-1} \cdots M_{2} \tilde{r}_{n}
$$

so by induction $\tilde{a}$ solves the complete system (11). Thus $a$ solves (10).

The construction we have used to solve (10) does not require the functions $r_{j}$ to be realvalued. In case they are real-valued, then $a$ and $g_{j}$ will be real-valued as well, and in Step 2 we may choose $U_{k}=\exp \left(i k^{-N} A_{N}\right)$, which is unitary. 
Step 4. - From steps 2 and 3 we obtain, for any positive integer $N$, an invertible operator $U_{N}=\left(U_{N, k}\right)_{\geqslant 0}$ (which is unitary in the case of self-adjoint operators $T_{j}$ ) and a smooth map

$$
g^{(N)}=\mathrm{Id}+k^{-1} g_{1}+\cdots+k^{-N} g_{N}
$$

such that

$$
U_{N}\left(T_{1}, \ldots, T_{n}\right) U_{N}^{-1}=g^{(N)}\left(T_{1}^{\Delta}, \ldots, T_{n}^{\Delta} ; k\right)+k^{-(N+1)} R_{N+1},
$$

where $R_{N+1}$ is a Toeplitz operator, and $U_{N}$ is of the form

$$
U_{N}=U^{(N)} U^{(N-1)} \cdots U^{(0)} \text {. }
$$

From step 3, we have

$$
U^{(j)}=\mathrm{Id}+\mathrm{i} k^{-j} A_{j} \bmod k^{-j} \mathscr{T},
$$

for $j \geqslant 1$. Therefore, one can construct by induction a sequence of symbols $\tilde{a}_{N}$ such that for all $N \geqslant 1$, the operator $U^{(N)} U^{(N-1)} \cdots U^{(1)}$ is, modulo $k^{-(N+1)} \mathscr{T}$, the Toeplitz quantization of the symbol

$$
1+\mathrm{i} k^{-1} \tilde{a}_{1}+\cdots+\mathrm{i} k^{-N} \tilde{a}_{N} .
$$

By the Borel summation procedure, one can find a Toeplitz operator $\tilde{A}$ whose total symbol has the asymptotic expansion

$$
\tilde{a}_{1}+k^{-1} \tilde{a}_{2}+\cdots+k^{-N+1} \tilde{a}_{N}+\cdots .
$$

Moreover, one can find a smooth map $\tilde{g}$ that admits the asymptotic expansion

$$
g^{(N)}=\mathrm{Id}+k^{-1} g_{1}+\cdots+k^{-N} g_{N}+\cdots .
$$

Now we let $\tilde{U}=\left(I+i k^{-1} \tilde{A}\right) U^{(0)}$, so that for any $N$,

$$
\tilde{U}=U_{N} \bmod k^{-(N+1)} \mathscr{T} \text {. }
$$

Thus from (14) we get, as required :

$$
\tilde{U}\left(T_{1}, \ldots, T_{n}\right) \tilde{U}^{-1}=\tilde{g}\left(T_{1}^{\Delta}, \ldots, T_{n}^{\Delta}\right)+\Theta\left(k^{-\infty}\right) .
$$

In the case where the operators $T_{j}$ are self-adjoint, one can change the construction of the sequence $\tilde{a}_{j}$ in such a way that $U^{(N)} U^{(N-1)} \cdots U^{(1)}$ is, modulo $k^{-(N+1)} \mathscr{T}$, the exponential of the Toeplitz quantization of the symbol $\mathrm{i} k^{-1} \tilde{a}_{1}+\cdots+\mathrm{i} k^{-N} \tilde{a}_{N}$. Then we define

$$
\tilde{U}:=\exp \left(\mathrm{i} k^{-1} \tilde{A}\right) U^{(0)},
$$

which is unitary.

\section{Isospectrality}

In this section we prove Theorem 1.1 and Corollary 1.2.

Recall that the joint spectrum of $n$ commuting matrices $A_{1}, \ldots, A_{n}$ is the set of

$$
\left(\lambda_{1}, \ldots, \lambda_{n}\right) \in \mathbb{C}^{n}
$$

such that there exists a non-zero vector $v$ for which

$$
A_{j} v=\lambda_{j} v
$$


for all $j=1, \ldots, n$. Such an $n$-uple $\left(\lambda_{1}, \ldots, \lambda_{n}\right)$ will be called a joint eigenvalue. We begin with the following elementary observations.

Lemma 6.1 ([14], Lemme 5.3). - Let $B_{1}, \ldots, B_{n}$ be commuting self-adjoint $k \times k$ matrices. Let $\epsilon>0$, and let $u \in \mathbb{C}^{k} \backslash\{0\}$ be such that $\left\|B_{i} u\right\| \leqslant \epsilon\|u\|$ for all $1 \leqslant i \leqslant n$. Then there exists $\lambda \in \mathbb{R}^{n}$ such that

$$
\lambda \in \operatorname{JointSpec}\left(B_{1}, \ldots, B_{n}\right) \cap[-\sqrt{n} \epsilon, \sqrt{n} \epsilon]^{n} .
$$

Proof. - Consider the matrix

$$
C:=\sqrt{B_{1}^{2}+\cdots+B_{n}^{2}}
$$

Since $C \geqslant 0$, the minimum of the spectrum of $C$ is equal to $\inf _{\|v\|=1}\|C v\|$. Since

$$
\forall v \in \mathbb{C}^{k}, \quad\|C v\|^{2}=\left\langle C^{2} v, v\right\rangle=\left\|B_{1} v\right\|^{2}+\cdots+\left\|B_{n} v\right\|^{2},
$$

we have $\|C u\| \leqslant \sqrt{n} \epsilon\|u\|$, thus $C$ admits an eigenvalue in $[0, \sqrt{n} \epsilon]$. The corresponding eigenspace being stable under the action of all $B_{j}$ 's, it contains a common eigenvector, and by (13), the corresponding joint eigenvalue $\left(\lambda_{1}, \ldots, \lambda_{n}\right)$ satisfies $\left|\lambda_{j}\right| \leqslant \sqrt{n} \epsilon$.

REMARK 6.2. - The constant factor $\sqrt{n}$ in Lemma 6.1 is optimal. Indeed, let $n$ and $k$ be integers with $1 \geqslant n \geqslant k$. For each $i$ with $1 \leqslant i \leqslant n$ consider the $k \times k$ diagonal matrix $B_{i}$ whose $j$ th column is equal to the zero vector for all $j \in\{1, \ldots, n\} \backslash\{i\}$, and equal to the canonical basis vector $\mathrm{e}_{j}$ otherwise. Let $u=\frac{\sum_{i=1}^{n} \mathrm{e}_{i}}{\sqrt{n}}$. Then $\|u\|^{2}=1$ and $B_{i} u=\frac{1}{\sqrt{n}} \mathrm{e}_{i}$, and hence $\left\|B_{i} u\right\|=\frac{1}{\sqrt{n}}$ for all $i=1, \ldots, n$. From Lemma 6.1 , the joint spectrum of $B_{1}, \ldots, B_{n}$ must contain a vector in $[-1,1]^{n}$. This bound is sharp: indeed, the joint spectrum is equal to the set $\left\{\mathrm{f}_{1}, \ldots, \mathrm{f}_{n}, \sum_{i=1}^{n} \mathrm{f}_{j}, \ldots, \sum_{i=1}^{n} \mathrm{f}_{j}\right\}$, where $\left(\mathrm{f}_{1}, \ldots, \mathrm{f}_{n}\right)$ is the canonical basis of $\mathbb{R}^{n}$ : thus its intersection with $[-t, t]^{n}$ is empty for any $0<t<1$.

Lemma 6.3. - The following statements hold.

(i) If $B_{1}, \ldots, B_{n}$ are commuting self-adjoint matrices, and $\alpha=\left(\alpha_{1}, \ldots, \alpha_{n}\right) \in \mathbb{C}^{n}$ is such that

$$
\left\|\left(B_{i}-\alpha_{i}\right) u\right\| \leqslant \epsilon\|u\|
$$

for all $1 \leqslant i \leqslant n$, then there exists a joint eigenvalue $\lambda \in \operatorname{JointSpec}\left(B_{1}, \ldots, B_{n}\right)$ such that $\left|\lambda_{i}-\alpha_{i}\right| \leqslant \sqrt{n} \epsilon$ for all $1 \leqslant i \leqslant n$.

(ii) Suppose that $A_{1}, \ldots, A_{n}$ is another collection of commuting self-adjoint matrices, and assume

$$
\left\|B_{i}-A_{i}\right\| \leqslant \epsilon \quad \text { for all } 1 \leqslant i \leqslant n .
$$

Then the Hausdorff distance between $\operatorname{JointSpec}\left(A_{1}, \ldots, A_{n}\right)$ and $\operatorname{JointSpec}\left(B_{1}, \ldots, B_{n}\right)$ is at most $\sqrt{n} \epsilon$, i.e.,

$$
\mathrm{d}_{H}\left(\operatorname{JointSpec}\left(A_{1}, \ldots, A_{n}\right), \operatorname{JointSpec}\left(B_{1}, \ldots, B_{n}\right)\right) \leqslant \sqrt{n} \epsilon .
$$

Proof. - The first statement is obtained from Lemma 6.1 applied to $B_{j}-\alpha_{j} I$. It implies that if $\alpha=\left(\alpha_{1}, \ldots, \alpha_{n}\right)$ is a joint eigenvalue of $\left(A_{1}, \ldots, A_{n}\right)$ and $\left\|B_{i}-A_{i}\right\| \leqslant \epsilon$ for all $1 \leqslant i \leqslant n$, then there exists a joint eigenvalue $\lambda \in \operatorname{JointSpec}\left(B_{1}, \ldots, B_{n}\right)$ with $\left|\lambda_{i}-\alpha_{i}\right| \leqslant \sqrt{n} \epsilon$ for all $1 \leqslant i \leqslant n$ (and vice-versa), which gives the last statement. 
If $T_{1}, \ldots, T_{n}$ are pairwise commuting Toeplitz operators, we call joint spectrum of $T_{1}, \ldots, T_{n}$ the sequence of joint spectra of the set of commuting matrices $\left(T_{1, k}, \ldots, T_{n, k}\right)$ acting on the Hilbert space $\mathscr{H}_{k}$.

Proof of Theorem 1.1. - By Theorem 5.2 applied to $\left(T_{1}, \ldots, T_{n}\right)$, we get an integer $k_{0}>0$, a sequence $(g(\cdot ; k))_{k \geqslant k_{0}}$ of smooth maps $\mathbb{R}^{n} \rightarrow \mathbb{R}^{n}$, and an operator $U=\left(U_{k}: \mathcal{H}_{k} \rightarrow \mathcal{H}_{k}^{\Delta}\right)_{k \geqslant k_{0}}$ with $U_{k}$ unitary, such that

$$
U_{k}\left(T_{1, k}, \ldots, T_{n, k}\right) U_{k}^{-1}=g\left(T_{1, k}^{\Delta}, \ldots, T_{n, k}^{\Delta} ; k\right)+\Theta\left(k^{-\infty}\right) .
$$

Let $S_{k}:=\operatorname{Joint} \operatorname{Spec}\left(T_{1}, \ldots, T_{n}\right)$. If we introduce the components of $g$,

$$
g=:\left(g_{1}, \ldots, g_{n}\right),
$$

then by Corollary 4.3, the joint spectrum of the commuting Toeplitz operators

$$
\left(g_{1}\left(T_{1, k}^{\Delta}, \ldots, T_{n, k}^{\Delta} ; k\right), \ldots, g_{n}\left(T_{1, k}^{\Delta}, \ldots, T_{n, k}^{\Delta} ; k\right)\right)
$$

is $\Sigma_{k}:=g\left(\left(v+\frac{2 \pi}{k} \mathbb{Z}^{n}\right) \cap \Delta, k\right)$.

Equation (14) means that there exists a sequence $\left(C_{N}\right)_{N \in \mathbb{N}}$ of real numbers such that

(15) for all $N$, for all $k \quad\left\|U_{k}\left(T_{1, k}, \ldots, T_{n, k}\right) U_{k}^{-1}-g\left(T_{1, k}^{\Delta}, \ldots, T_{n, k}^{\Delta} ; k\right)\right\| \leqslant C_{N} k^{-N}$.

Then, by virtue of Lemma 6.3, we have

$$
\text { for all } N, \text { for all } k, \quad \mathrm{~d}_{H}\left(S_{k}, \Sigma_{k}\right) \leqslant \sqrt{n} C_{N} k^{-N}
$$

which means by definition $S_{k}=\Sigma_{k}+\Theta\left(k^{-\infty}\right)$ as we wanted to show.

Now we prove that for all sufficiently large $k$, the multiplicity of the eigenvalues of JointSpec $\left(T_{1}, \ldots, T_{n}\right)$ is 1 , and there exists a small constant $\delta>0$ such that each

ball of radius $\frac{\delta}{k}$ centered at an eigenvalue contains precisely only that eigenvalue. First we observe that this statement holds when instead of $\operatorname{Joint} \operatorname{Spec}\left(T_{1}, \ldots, T_{n}\right)$ we consider $\Sigma_{k}$. The multiplicity one statement is a direct consequence of Theorem 4.1, and the separation property follows from the fact that the principal term of $g$ is the identity map.

Consider $\lambda=\lambda(k) \in S_{k} \subseteq \mathbb{R}^{n}$ and the self-adjoint operators

$$
C_{k}:=\sqrt{\left(T_{1, k}-\lambda_{1} \operatorname{Id}_{k}\right)^{2}+\cdots+\left(T_{n, k}-\lambda_{n} \operatorname{Id}_{k}\right)^{2}}
$$

and

$$
C_{k}^{\Delta}:=\sqrt{\left(T_{1, k}^{\Delta}-\lambda_{1} \operatorname{Id}_{k}\right)^{2}+\cdots+\left(T_{n, k}^{\Delta}-\lambda_{n} \operatorname{Id}_{k}\right)^{2}}
$$

Let

$$
0=\lambda_{1}\left(C_{k}\right) \leqslant \lambda_{2}\left(C_{k}\right) \leqslant \cdots \leqslant \lambda_{d_{k}}\left(C_{k}\right)
$$

be the eigenvalues of $C_{k}$ repeated with multiplicity, where $d_{k}$ is the dimension of $\mathcal{H}_{k}$. Let

$$
\lambda_{1}\left(C_{k}^{\Delta}\right) \leqslant \lambda_{2}\left(C_{k}^{\Delta}\right) \leqslant \cdots \leqslant \lambda_{d_{k}}\left(C_{k}^{\Delta}\right)
$$

be the eigenvalues of $C_{k}^{\Delta}$ repeated with multiplicity. By the min-max formula we have that for any $j=1, \ldots, d_{k}$,

$$
\left|\lambda_{j}\left(C_{k}\right)-\lambda_{j}\left(C_{k}^{\Delta}\right)\right|=\left|\lambda_{j}\left(U_{k} C_{k} U_{k}^{-1}\right)-\lambda_{j}\left(C_{k}^{\Delta}\right)\right| \leqslant\left\|U_{k} C_{k} U_{k}^{-1}-C_{k}^{\Delta}\right\| \leqslant \frac{M}{k^{2}}
$$


for some constant $M>0$. Therefore for any $j>1$ the inequality $\left|\lambda_{j}\left(C_{k}^{\Delta}\right)-\lambda_{1}\left(C_{k}^{\Delta}\right)\right| \geqslant \frac{\delta}{k}$ implies

$$
\left|\lambda_{j}\left(C_{k}\right)\right|=\left|\lambda_{j}\left(C_{k}\right)-\lambda_{1}\left(C_{k}\right)\right| \geqslant \frac{\delta}{k}-\frac{2 M}{k^{2}} \geqslant \frac{\delta^{\prime}}{k}
$$

for some $\delta^{\prime}>0$. Thus 0 as an eigenvalue of $C_{k}$ has multiplicity one, and is the only eigenvalue of $C_{k}$ in the interval $\left[-\frac{\delta^{\prime}}{k}, \frac{\delta^{\prime}}{k}\right]$. Going back to the commuting operators $T_{1}, \ldots, T_{n}$, we see that the joint eigenvalue $\lambda \in S_{k}$ has multiplicity one, and all other joint eigenvalues lie outside of the Euclidean ball centered at $\lambda$ of radius $\frac{\delta^{\prime}}{k}$.

Proof of Corollary 1.2. - Recall that we defined the limit of a sequence $\left(\mathscr{Q}_{k}\right)_{k \in \mathbb{N}}$ of subsets of $\mathbb{R}^{n}$ by

$$
\lim \mathscr{G}_{k}:=\left\{c \in \mathbb{R}^{n} \mid \forall U \text { neighborhood of } c, \exists k_{0} \text { such that } \forall k \geq k_{0}, U \cap \mathscr{C}_{k} \neq \varnothing\right\} .
$$

We denote by $\mathrm{B}(c, r)$ the open ball in $\mathbb{R}^{n}$ centered at $c$ and of radius $r$. Notice that if $\mathscr{Q}_{k} \subset \mathscr{B}_{k}+\mathrm{B}\left(0, C k^{-1}\right)$, for some constant $C$, then

$$
\lim \mathscr{G}_{k} \subset \lim \mathscr{B}_{k} .
$$

We use the same notation as in the proof of Theorem 1.1: $S_{k}=\operatorname{JointSpec}\left(T_{1}, \ldots, T_{n}\right)$ and

$$
\Sigma_{k}=g\left(\left(v+\frac{2 \pi}{k} \mathbb{Z}^{n}\right) \cap \Delta, k\right) .
$$

Step 1. - The estimate (15) for $N=1$ gives

$$
S_{k} \subset \Sigma_{k}+\mathrm{B}\left(0, C k^{-1}\right) \quad \text { and } \quad \Sigma_{k} \subset S_{k}+\mathrm{B}\left(0, C k^{-1}\right) .
$$

Therefore, $\lim S_{k}=\lim \Sigma_{k}$.

Step 2. - Now let us show that $\lim \Sigma_{k}=\Delta$. From Theorem 5.2, we know that $g$ admits an asymptotic expansion in the $\mathrm{C}^{\infty}$ topology of the form

$$
g(\cdot ; k)=\mathrm{Id}+k^{-1} g_{1}+k^{-2} g_{2}+\cdots .
$$

Therefore, for any compact $K \subset \mathbb{R}^{n}$, there exists a constant $C$ such that

$$
\max _{c \in K}\|g(c ; k)-c\|_{\mathbb{R}^{n}} \leqslant C k^{-1} .
$$

We may choose $K$ large enough so that it contains $\Delta$, and we get the following inclusions :

$$
\Sigma_{k} \subset \Delta \cap\left(v+\frac{2 \pi}{k} \mathbb{Z}^{n}\right)+\mathrm{B}\left(0, C k^{-1}\right) \text { and } \Delta \cap\left(v+\frac{2 \pi}{k} \mathbb{Z}^{n}\right) \subset \Sigma_{k}+\mathrm{B}\left(0, C k^{-1}\right) .
$$

Hence

$$
\lim \Sigma_{k}=\lim \Delta \cap\left(v+\frac{2 \pi}{k} \mathbb{Z}^{n}\right)=\Delta .
$$

From steps 1 and 2 we conclude that

$$
\lim S_{k}=\Delta
$$

which finishes the proof.

Corollary 6.4 (Isospectrality). - Two symplectic toric systems are isomorphic if and only if the limit of their joint spectra coincide. In particular, if two symplectic toric systems have the same joint spectra, then they are isomorphic. 


\section{Metaplectic correction}

Theorem 1.1 and Theorem 1.2 can be proven analogously to our proofs in the presence of a half form bundle. Below we explain the corresponding modifications needed.

Toeplitz quantization is often considered more natural in the presence of a half-form bundle. Let $(M, \omega, j)$ be a compact Kähler manifold. A half-form bundle of $(M, j)$ is a square root of the canonical bundle of $M$. More precisely we consider a pair $(\delta, \varphi)$ consisting of a complex line bundle $\delta \rightarrow M$ and an isomorphism

$$
\varphi: \delta^{\otimes 2} \rightarrow \wedge^{n, 0} \mathrm{~T}^{*} M
$$

Here $n$ is the complex dimension of $M$. Such a square root does not necessarily exist, and if it exists, the space of half-form bundles up to isomorphism is a principal homogeneous space for the group $\mathrm{H}^{1}(M, \mathbb{Z} / 2 \mathbb{Z})$.

Let $\mathcal{L} \rightarrow M$ be a prequantum bundle and $(\delta, \varphi)$ be a half-form bundle. Observe that $\delta$ has a metric and a holomorphic structure determined by the condition that $\varphi$ is an isomorphism of Hermitian holomorphic bundles. Define the quantum space $\mathcal{H}_{\mathrm{m}, k}$ as the vector space of holomorphic sections of $\mathcal{L}^{k} \otimes \delta$. The space $\mathcal{H}_{\mathrm{m}, k}$ has a natural scalar product obtained by integrating the pointwise scalar product of sections of $\mathcal{L}^{k} \otimes \delta$ against the Liouville measure. This scalar product is actually defined on the space of $\mathrm{L}^{2}$ sections, and we have an orthogonal projector $\Pi_{k}$ from the space of $\mathrm{L}^{2}$ sections onto $\mathcal{H}_{\mathrm{m}, k}$. The definition of Toeplitz operator is the same as before except that we use this new projector. So a Toeplitz operator is any family $\left(T_{k}: \mathcal{H}_{\mathrm{m}, k} \rightarrow \mathcal{H}_{\mathrm{m}, k}\right)_{k \in \mathbb{N}^{*}}$ of endomorphisms of the form

$$
T_{k}=\Pi_{k} f(\cdot, k)+R_{k}, \quad k \in \mathbb{N}^{*}
$$

where $f(\cdot, k)$, viewed as a multiplication operator, is a sequence in $\mathrm{C}^{\infty}(M)$ with an asymptotic expansion

$$
f_{0}+k^{-1} f_{1}+\cdots
$$

for the $\mathrm{C}^{\infty}$ topology, and the norm of $R_{k}$ is $\theta\left(k^{-\infty}\right)$. Theorem 5.1 still holds and we define the principal and subprincipal symbols of a Toeplitz operator with the same formula as before. The rule of composition of these symbols is still given by (18). We can also define Toeplitz operators by using the Kostant-Souriau formula. Consider a smooth function $f$ on $M$ whose Hamiltonian vector field $X$ preserves the complex structure. Then the following operators are well defined

$$
T_{k}=f+\frac{1}{\mathrm{i} k}\left(\nabla_{X}^{\varphi^{k}} \otimes \mathrm{Id}+\mathrm{Id} \otimes \mathrm{L}_{X}^{\delta}\right): \mathcal{H}_{\mathrm{m}, k} \rightarrow \mathcal{H}_{\mathrm{m}, k}, \quad k \in \mathbb{N}^{*} .
$$

Here $\mathrm{L}^{\delta}$ is the Lie derivative of the half-form, that is the first order differential operator such that for any local section $s$ of the half-form bundle one has

$$
\mathrm{L}_{X}\left(\varphi\left(s^{\otimes 2}\right)\right)=2 \varphi\left(s \otimes \mathrm{L}_{X}^{\delta} s\right) .
$$

One also shows that the family $\left(T_{k}\right)$ is a Toeplitz operator with principal symbol $f$ and vanishing subprincipal symbol.

Consider now the Delzant space $M_{\Delta}$ defined as in Section 2. Since $M_{\Delta}$ is simply connected, there exists at most one half-form bundle. 
Recall that we denote by $F$ the set of faces of $\Delta$ of codimension 1 and for each $f \in F$, $X_{f} \in \mathfrak{t}$ is the primitive normal vector to the face.

Proposition 7.1. - The Delzant space $M_{\Delta}$ admits an equivariant half-form bundle if and only if there exists $\gamma \in \mathfrak{t}_{\mathbb{Z}}^{*}$ such that $\gamma\left(X_{f}\right)$ is odd for any $f \in F$.

Proof. - This criterion may be established using the divisor of the toric variety, cf. [17]. To each face $f \in F$ corresponds an irreducible divisor $D_{f}$ of $M_{\Delta}$. It is known that a divisor of the canonical bundle is $-\sum_{f \in F} D_{f}$. Recall also that the divisors $D_{f}$ 's generate the Picard group, and that $\sum n_{f} X_{f}$ is principal if and only if $n_{f}=\gamma\left(X_{f}\right)$ for some $\gamma \in \mathfrak{t}_{\mathbb{Z}}^{*}$. So $M_{\Delta}$ admits a half-form bundle if and only if there exists a divisor

$$
D=\sum n_{f}^{\delta} D_{f}
$$

such that $2 D+\sum_{f \in F} D_{f}$ is principal, that is

$$
2 n_{f}^{\delta}+1=\left\langle X_{f}, \gamma\right\rangle
$$

for some $\gamma \in \mathfrak{t}_{\mathbb{Z}}^{*}$.

Assume now that the vertices of $\Delta$ belongs to $2 \pi \mathfrak{t}_{\mathbb{Z}}^{*}$ so that $\left(M_{\Delta}, \omega_{M_{\Delta}}\right)$ admits a prequantum bundle $\mathscr{L}_{\Delta}$ unique up to isomorphism. Assume also that $M_{\Delta}$ is equipped with a halfform bundle $\delta_{\Delta}$. For any positive integer $k$, define the quantum space

$$
\mathcal{H}_{\mathrm{m}, k}^{\Delta}=\mathrm{H}^{0}\left(M_{\Delta}, \mathscr{L}_{\Delta}^{k} \otimes \delta_{\Delta}\right) .
$$

For any $X \in \mathfrak{t}$, consider the rescaled Kostant-Souriau operators

$$
T_{X, k}:=\left\langle\mu_{\Delta}, X\right\rangle+\frac{1}{\mathrm{i} k}\left(\nabla_{X^{\sharp}} \otimes \mathrm{Id}+\mathrm{Id} \otimes \mathrm{L}_{X}^{\delta}\right): \mathcal{H}_{\mathrm{m}, k}^{\Delta} \rightarrow \mathcal{H}_{\mathrm{m}, k}^{\Delta} .
$$

We can now state the analogue of Theorem 4.1. Introduce $\gamma \in \mathfrak{t}_{\mathbb{Z}}^{*}$ such that $\gamma\left(X_{f}\right)$ is odd for any $f \in F$.

THEOREM 7.2. - There is an orthogonal decomposition of the quantum space $\mathcal{H}_{\mathrm{m}, k}^{\Delta}$ into a direct sum of lines:

$$
\mathcal{H}_{\mathrm{m}, k}^{\Delta}=\bigoplus_{\ell \in\left(\frac{2 \pi}{k}\left(\mathfrak{t}_{\mathbb{Z}}^{*}+\frac{1}{2} \gamma\right)\right) \cap \Delta} D_{\ell}^{k}
$$

such that, for any $X \in \mathfrak{t}$,

$$
T_{X, k} \Psi=\ell(X) \Psi, \quad \text { for all } \Psi \in D_{\ell}^{k} .
$$

Observe that the points of $\left(\frac{2 \pi}{k}\left(\mathfrak{t}_{\mathbb{Z}}^{*}+\frac{1}{2} \gamma\right)\right) \cap \Delta$ are all in the interior of $\Delta$. Furthermore on a neighborhood of each vertex we recover the usual joint spectrum of $n$ harmonic oscillators as follows. For any vertex $v$ denote by $F_{v}$ the set of one-codimensional faces adjacent to $v$ so that $\left(f_{v}, v \in F_{v}\right)$ is a basis of $t_{\mathbb{Z}}^{*}$. Then there exists a neighborhood $U$ of $v$ such that

$$
\Delta \cap U=\left\{v+x /\langle f, x\rangle \geqslant 0, \forall f \in F_{v}\right\} \cap U
$$

and

$$
\left(\frac{2 \pi}{k}\left(\mathfrak{t}_{\mathbb{Z}}^{*}+\frac{1}{2} \gamma\right)\right) \cap \Delta \cap U=\left\{v+x /\langle f, x\rangle \in \frac{2 \pi}{k}\left(\mathbb{N}+\frac{1}{2}\right), \forall f \in F_{v}\right\} \cap U .
$$

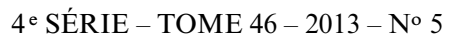


Proof. - Let us adapt the proof of Theorem 4.1. First we introduce the quantization of $\mathbb{C}^{F}$ with metaplectic correction. Choose an ordering of $F$ and define $v \in \Omega\left(\mathbb{C}^{F}\right)$ as the wedge product of the $\mathrm{d} z_{f}$ 's. $v$ is a non vanishing section of the canonical bundle of $\mathbb{C}^{F}$. The action of $t \in \mathbb{R}^{F} / \mathbb{Z}^{F}$ on the canonical bundle sends $v$ into $\exp \left(-2 \mathrm{i} \pi \sum t_{f}\right) v$.

Let $\delta_{F}$ be the trivial complex line bundle with base $\mathbb{C}^{F}$ and $\varphi_{F}$ be the isomorphism from $\delta_{F}^{2}$ to the canonical bundle of $\mathbb{C}^{F}$ given by $\varphi(z, 1)=v(z)$. By Proposition 7.1 , there exist $d \in \mathbb{Z}^{F}$ and $\gamma \in \mathfrak{t}_{\mathbb{Z}}^{*}$ such that

$$
\pi^{*} \gamma=\mathbb{I}+2 d
$$

where $\mathbb{I}$ is the vector in $\left(\mathbb{R}^{F}\right)^{*}$ with all components equal to 1 . Consider the action of $\mathbb{R}^{F} / \mathbb{Z}^{F}$ on $\delta_{F}$ given by

$$
t \cdot(z, u)=\left(t \cdot z, e^{2 \mathrm{i} \pi \sum t_{f} d_{f}} u\right)
$$

Then the isomorphism $\varphi$ intertwines the action of the subtorus $N$ of $\mathbb{R}^{F} / \mathbb{Z}^{F}$ on $\delta_{F}$ with the action of $N$ on the canonical bundle. This condition has the consequence that the quotient of $\delta_{F}^{2}$ by $N$ defines a half-form bundle $\delta_{\Delta}$ on the Delzant space $M_{\Delta}$, cf. Section 8.3 of [12]. Furthermore one has an isomorphism

$$
\Phi_{k}:\left(\mathcal{B}_{\mathrm{m}, k}\right)^{N} \rightarrow \mathcal{H}_{\mathrm{m}, k}^{\Delta}
$$

from the $N$-invariant part of the space $\mathscr{B}_{\mathrm{m}, k}$ of holomorphic sections of $\mathcal{L}_{F}^{k} \otimes \delta_{F}$ to the quantum space $\mathcal{H}_{\mathrm{m}, k}^{\Delta}$.

Introduce the rescaled Kostant-Souriau operators

$$
S_{X, k}:=\langle\tilde{\mu}, X\rangle+\frac{1}{\mathrm{i} k}\left(\nabla_{X^{\sharp}}^{\varphi^{k}} \otimes \operatorname{Id}+\operatorname{Id} \otimes \mathrm{L}_{X^{\sharp}}^{\delta}\right) .
$$

Then $\left(\mathcal{B}_{\mathrm{m}, k}\right)^{N}$ consists of the sections $\Psi \in \mathscr{B}_{\mathrm{m}, k}$ such that $S_{X, k} \Psi=0$ for any $X \in \mathfrak{n}$. Furthermore

$$
\Phi_{k}\left(S_{X, k} \Psi\right)=T_{\pi(X), k} \Phi_{k}(\Psi), \quad \text { for all } \Psi \in\left(\mathcal{B}_{\mathrm{m}, k}\right)^{N} .
$$

To conclude the proof let us compute the action of the rescaled Kostant-Souriau operators. We have for $X=e_{f}$

$$
S_{X, k}\left(\mathrm{e}^{-\frac{k}{8 \pi}|z|^{2}} g(z)\right)=\mathrm{e}^{-\frac{k}{8 \pi}|z|^{2}}\left(\frac{2 \pi}{k}\left(z_{f} \partial_{z_{f}} g+\frac{1}{2} g\right)-\lambda_{f} g\right) .
$$

For any $\alpha \in \mathbb{N}^{F}$ set $\psi_{\alpha}=\mathrm{e}^{-\frac{k}{2}|z|^{2}} z^{\alpha}$ so that

$$
S_{X, k}\left(\psi_{\alpha}\right)=\left\langle X, \frac{2 \pi}{k}\left(\alpha+\frac{1}{2} \mathbb{I}\right)-\lambda\right\rangle \psi_{\alpha} .
$$

Hence the space $\left(\mathscr{B}_{\mathrm{m}, k}\right)^{N}$ admits as a basis the family $\left(\Psi_{\alpha}\right)$ where $\alpha$ runs over $\left(\mathbb{Z}^{F}+\frac{1}{2} \mathbb{I}\right) \cap \operatorname{ker} \iota_{\mathfrak{n}}^{*}$. To conclude we prove as in Lemma 4.2 that $\pi^{*}+\lambda$ restricts to a bijection

$$
\frac{2 \pi}{k}\left(\mathfrak{t}_{\mathbb{Z}}^{*}+\frac{1}{2} \gamma\right) \longrightarrow \frac{2 \pi}{k}\left(\mathbb{Z}^{F}+\frac{1}{2} \mathbb{I}\right) \cap \operatorname{ker} \iota_{\mathfrak{n}}^{*} .
$$

Let us consider now the generalization of Theorem 5.2. Let $\left(M, \omega, \mathbb{R}^{n} / \mathbb{Z}^{n}, \mu\right)$ be a symplectic toric manifold equipped with a prequantum bundle $\mathcal{L}$, a compatible complex structure $j$ and a half-form bundle $\delta$. Denote by $\Delta$ the momentum polytope $\mu(M) \subset \mathbb{R}^{n}$. Let $T_{1}, \ldots, T_{n}$ be commuting self-adjoint Toeplitz operators of $\mathcal{H}_{\mathrm{m}, k}$ whose principal symbols are the components of $\mu$. Denote by $f_{1}^{i}$ the subprincipal symbol of $T_{i}$. 


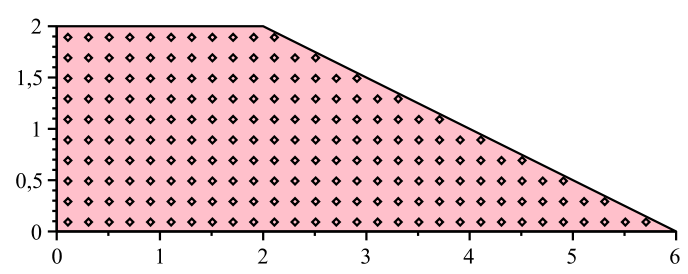

FIGURE 5. Spectrum in Figure 2 with metaplectic correction.

THEOREM 7.3. - There exists $k_{0}>0$, there exists a sequence $(g(\cdot ; k))_{k \geqslant k_{0}}$ of smooth maps $\mathbb{R}^{n} \rightarrow \mathbb{R}^{n}$, and there exists an operator $U=\left(U_{k}: \mathcal{H}_{k} \rightarrow \mathcal{H}_{k}^{\Delta}\right)_{k \geqslant k_{0}}$ with $U_{k}$ unitary for any $k$, such that

$$
U_{k}\left(T_{1, k}, \ldots, T_{n, k}\right) U_{k}^{-1}=g\left(T_{1, k}^{\Delta}, \ldots, T_{n, k}^{\Delta} ; k\right)+\Theta\left(k^{-\infty}\right) .
$$

Moreover, $g$ admits an asymptotic expansion in the $\mathrm{C}^{\infty}$ topology of the form $\mathrm{Id}+k^{-1} g_{1}+$ $k^{-2} g_{2}+\cdots$ where the subprincipal term $g_{1}$ is given by

$$
g_{1}^{i}(E)=\int_{1}^{0} f_{1}^{i}\left(\varphi_{\mu_{i}}^{t}(x)\right) \mathrm{d} t, \quad \text { for all } E \in \Delta, x \in \mu^{-1}(E) .
$$

Here $\varphi_{\mu_{i}}^{t}$ is the Hamiltonian flow of $\mu_{i}$.

Proof. - The proof is the same as the one of Theorem 5.2. Because of the metaplectic correction, we can choose in the first step the operator $U_{k}$ quantizing $\varphi$ in such a way that for any Toeplitz operator $\left(S_{k}\right),\left(S_{k}\right)$ and $\left(U_{k} S_{k} U_{k}^{*}\right)$ have the same principal and subprincipal symbols. This follows from Theorem 5.1 in [11]. From this we can extract $g_{1}$ from the cohomological equation

$$
\left\{\mu_{i}, a\right\}=f_{1}^{i}-g_{1}^{i}\left(\mu_{\Delta}\right) .
$$

Given the statement of Theorem 7.3 above, the proof of Theorem 1.3 is identical to the proof of Theorem 1.1 given in Section 6 .

\section{Final Remarks}

\section{Isospectrality in geometry}

In the present paper we have dealt with isospectrality in the context of integrable systems and symplectic geometry. The paper settles the Spectral Goal for Quantum Systems for the case of toric systems outlined in the last two authors' article [60]: to prove that large classes of integrable systems are determined by their semiclassical joint spectrum.

Corollary 1.2 says that the joint spectrum does indeed determine the system. This type of conclusion often has a negative answer, at least if one considers it in Riemannian geometry. In Riemannian geometry the operator whose spectrum is considered is the Laplace operator. Two compact Riemannian manifolds are said to be isospectral if the associated Laplace operators have the same spectrum.

Bochner and Kac's question has a negative answer in this case, even for planar domains with Dirichlet boundary conditions (which is the original version posed in [41]). As P. Sarnak 
mentioned to us, a much better question to ask is whether the set of isospectral domains is finite. There are many related works, see for instance Milnor [52], Sunada [64], OsgoodPhillips-Sarnak [54, 55, 56], Bérard [2], Buser [4] and Gordon-Webb-Wolpert [32].

As we have mentioned, in symplectic geometry a few positive results are known. These results, and the present paper, give evidence that symplectic invariants seem to be more encodable in the spectrum of a quantum integrable system than Riemannian invariants in the spectrum of the Lapace operator.

Inverse type results in the realm of spectral geometry have been obtained by many other authors, see for instance Brüning-Heintze [3], Colin de Verdière [14, 13, 15], Colin de Verdière-Guillemin [16], Croke-Sharafutdinov [18], Guillemin-Kazhdan [35], McKeanSinger [50], Osgood-Phillips-Sarnak [54, 55, 56], and Zelditch [75], and the references therein. An interesting general problem (for instance in the context of toric geometry) is to what extent information about measures may be recovered from the spectrum, see Guillemin-Sternberg [38, p. 268-271] for a result in this direction.

\section{Isospectral conjecture for semitoric systems}

In $[61,59]$ the last two authors formulated an inverse spectral conjecture for semitoric completely integrable systems (see [57, 58] for a classification of semitoric systems in terms of five symplectic invariants): the semiclassical joint spectrum of a quantum semitoric system determines the corresponding classical system.

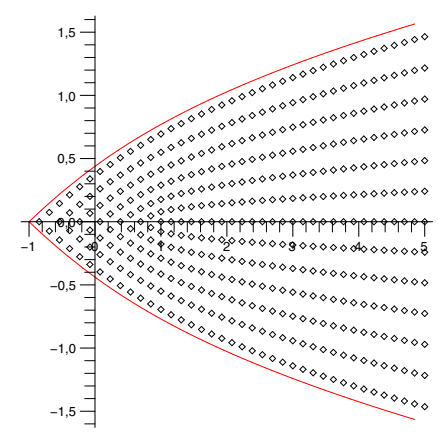

FIgURE 6. The quantum coupled spin-oscillator is a fundamental example of quantum semitoric integrable system. Its joint spectrum is depicted in the figure for a fixed value of the spectral parameter.

Semitoric systems are four-dimensional integrable systems with two degrees of freedom for which one component of the system generates a $2 \pi$-periodic flow. Semitoric systems lie somewhere in between toric systems and general integrable systems. If both components of the semitoric system are $2 \pi$-periodic, i.e., the system is generated by a Hamiltonian 2 -torus action, then the system is a toric system (strictly speaking after a harmless rescaling of the periods).

Theorem 1.2 above solves the conjecture for the class of toric systems. In this class the result is even stronger, since there is no restriction on the dimension, and moreover only the 
spectrum modulo $\theta(\hbar)$ is needed, whereas in general one expects that an accuracy of order $\theta\left(\hbar^{2}\right)$ is necessary.

\section{Microlocal analysis of integrable systems}

The notion of a quantum integrable system, as a maximal set of commuting quantum observables, dates back to the early quantum mechanics, to the works of Bohr, Sommerfeld and Einstein [29]. However, the most basic results in the symplectic theory of classical integrable systems like Darboux's theorem or action-angle variables could not be used in Schrödinger's quantum setting at that time because they make use of the analysis of differential (or pseudodifferential) operators in phase space, known now as microlocal analysis, which was developed only in the 1960s. The microlocal analysis of action-angle variables starts with the works of Duistermaat [23] and Colin de Verdière [14, 13], followed by the semiclassical theory by Charbonnel [5], and more recently by Vũ Ngọc [69], Zelditch and Toth [65, 66, 67], Charbonnel and Popov [6], Melin-Sjöstrand [51], and many others.

Effective models in quantum mechanics often require a compact phase space, and thus cannot be treated using pseudodifferential calculus. For instance the natural classical limit of a quantum spin is a symplectic sphere. The study of quantum action-angle variables in the case of compact symplectic manifolds treated in this paper was started by Charles [8], using the theory of Toeplitz operators. In the present paper, we present global spectral results for toric integrable systems; we use in a fundamental way Delzant's theorem on symplectic toric manifolds [21].

Toric integrable systems ${ }^{(2)}$ have played an influential role in symplectic and complex algebraic geometry, representation theory, and spectral theory since T. Delzant classified them in terms of combinatorial information (actually, in terms of a convex polytope, see Theorem 2.3). A comparative study of symplectic toric manifolds from the symplectic and complex algebraic viewpoints was given by Duistermaat and Pelayo [28]. A beautiful treatment of the classical theory of toric systems is given in Guillemin's book [34].

Toeplitz operators are a natural generalization of Toeplitz matrices (which correspond to Toeplitz operators on the unit disk). On $\mathbb{R}^{n}$, Toeplitz operators correspond to pseudodifferential operators via the Bargmann transform. Of course, such a correspondence cannot hold in the case of a compact phase space, but it turns out that Toeplitz operators always give rise to a semiclassical algebra of operators with a symbolic calculus and microlocalization properties, which is microlocally isomorphic to the algebra of pseudodifferential operators. See the book by Boutet de Monvel and Guillemin for an introduction to the spectral theory of Toeplitz operators [53].

(2) Toric integrable systems always have singularities of elliptic and transversally elliptic type, but do not have singularities of hyperbolic or focus-focus type. The local and semiglobal theory for regular points, elliptic and transversally elliptic singularities is now well understood both at the symplectic level (action-angle theorem of Liouville-Arnold-Mineur, Eliasson's linearization theorems), as well as the quantum level, see Charles [8] and Vũ Ngọc [70]. This gives the foundation of the modern theory of integrable systems, in the spirit of Duistermaat's article [24], but also of KAM-type perturbation theorems (see de la Llave's survey article [48]).

$4^{\text {e }}$ SÉRIE - TOME $46-2013$ - No 5 


\section{Acknowledgements}

We are very grateful to a referee for many insightful comments which have improved the paper, in particular for pointing out an omission of a constant in one of the lemmas (Lemma 6.1) which affected the constants in Lemma 6.3.

We thank Jochen Brüning, Helmut Hofer, Peter Sarnak, and Thomas Spencer for fruitful discussions. The authors are grateful to Helmut Hofer for his essential support, which made it possible for LC and VNS to visit AP at the Institute for Advanced Study during the Winter and Summer of 2011, where a part of this paper was written. Additional financial support for the visits was provided by Washington University and NSF.

AP was partly supported by an NSF Postdoctoral Fellowship, a MSRI membership, an IAS membership, NSF Grants DMS-0965738 and DMS-0635607, an NSF CAREER Award, a Leibniz Fellowship from the Mathematisches Forschungsinstitut Oberwolfach, Spanish Ministry of Science Grant MTM 2010-21186-C02-01, and by the Spanish National Research Council (CSIC). VNS was partly supported by the NONA, a grant from the French ANR and the Institut Universitaire de France.

\section{REFERENCES}

[1] M. F. Atryah, Convexity and commuting Hamiltonians, Bull. London Math. Soc. 14 (1982), 1-15.

[2] P. BÉRARd, Transplantation et isospectralité. I, Math. Ann. 292 (1992), 547-559.

[3] J. Brüning, E. Heintze, Spektrale Starrheit gewisser Drehflächen, Math. Ann. 269 (1984), 95-101.

[4] P. Buser, Isospectral Riemann surfaces, Ann. Inst. Fourier (Grenoble) 36 (1986), 167192.

[5] A.-M. Charbonnel, Comportement semi-classique du spectre conjoint d'opérateurs pseudodifférentiels qui commutent, Asymptotic Anal. 1 (1988), 227-261.

[6] A.-M. Charbonnel, G. Popov, A semi-classical trace formula for several commuting operators, Comm. Partial Differential Equations 24 (1999), 283-323.

[7] L. Charles, Berezin-Toeplitz operators, a semi-classical approach, Comm. Math. Phys. 239 (2003), 1-28.

[8] L. Charles, Quasimodes and Bohr-Sommerfeld conditions for the Toeplitz operators, Comm. Partial Differential Equations 28 (2003), 1527-1566.

[9] L. Charles, Symbolic calculus for Toeplitz operators with half-form, J. Symplectic Geom. 4 (2006), 171-198.

[10] L. Charles, Toeplitz operators and Hamiltonian torus actions, J. Funct. Anal. 236 (2006), 299-350.

[11] L. Charles, Semi-classical properties of geometric quantization with metaplectic correction, Comm. Math. Phys. 270 (2007), 445-480. 
[12] L. Charles, On the quantization of polygon spaces, Asian J. Math. 14 (2010), 109-152.

[13] Y. Colin de Verdière, Spectre conjoint d'opérateurs pseudo-différentiels qui commutent. I. Le cas non intégrable, Duke Math. J. 46 (1979), 169-182.

[14] Y. Colin de Verdière, Spectre conjoint d'opérateurs pseudo-différentiels qui commutent. II. Le cas intégrable, Math. Z. 171 (1980), 51-73.

[15] Y. Colin de Verdière, A semi-classical inverse problem II: reconstruction of the potential, in Geometric aspects of analysis and mechanics, Progr. Math. 292, Birkhäuser, 2011, 97-119.

[16] Y. Colin de Verdière, V. Guillemin, A semi-classical inverse problem I: Taylor expansions, in Geometric aspects of analysis and mechanics, Progr. Math. 292, Birkhäuser, 2011, 81-95.

[17] D. A. Cox, J. B. Little, H. K. Schenck, Toric varieties, Graduate Studies in Math. 124, Amer. Math. Soc., 2011.

[18] C. B. Croke, V. A. Sharafutdinov, Spectral rigidity of a compact negatively curved manifold, Topology 37 (1998), 1265-1273.

[19] V. I. Danilov, The geometry of toric varieties, Uspekhi Mat. Nauk 33 (1978), 85-134, 247.

[20] K. Datchev, H. Hezari, I. Ventura, Spectral uniqueness of radial semiclassical Schrödinger operators, Math. Res. Lett. 18 (2011), 521-529.

[21] T. Delzant, Hamiltoniens périodiques et images convexes de l'application moment, Bull. Soc. Math. France 116 (1988), 315-339.

[22] E. B. Dryden, V. Guillemin, R. Sena-Dias, Hearing Delzant polytopes from the equivariant spectrum, to appear in Trans. Amer. Math. Soc.

[23] J. J. Duistermant, Oscillatory integrals, Lagrange immersions and unfolding of singularities, Comm. Pure Appl. Math. 27 (1974), 207-281.

[24] J. J. Duistermant, On global action-angle coordinates, Comm. Pure Appl. Math. 33 (1980), 687-706.

[25] J. J. Duistermant, Principal fiber bundles, in Notes for the Utrecht Spring School, 2004, http://www.projects.science.uu.nl/Duistermaat/www/homepageHD/ pf.pdf.

[26] J. J. Duistermant, The heat kernel Lefschetz fixed point formula for the spin-c Dirac operator, Modern Birkhäuser Classics, Birkhäuser, 2011.

[27] J. J. Duistermaat, L. Hörmander, Fourier integral operators. II, Acta Math. 128 (1972), 183-269.

[28] J. J. Duistermaat, Á. Pelayo, Reduced phase space and toric variety coordinatizations of Delzant spaces, Math. Proc. Cambridge Philos. Soc. 146 (2009), 695-718.

[29] A. Einstein, Zum Quantensatz von Sommerfeld und Epstein, Verhandlungen der Deutschen Physikalischen Gesellschaft 19 (1917), 82-92.

[30] L. H. Eliasson, Normal forms for Hamiltonian systems with Poisson commuting integrals - elliptic case, Comment. Math. Helv. 65 (1990), 4-35.

[31] M. D. Garay, D. van Straten, Classical and quantum integrability, Mosc. Math. J. 10 (2010), 519-545, 661.

$4^{\mathrm{e}}$ SÉRIE - TOME $46-2013$ - No 5 
[32] C. Gordon, D. L. Webb, S. Wolpert, Isospectral plane domains and surfaces via Riemannian orbifolds, Invent. Math. 110 (1992), 1-22.

[33] C. Gordon, D. L. Webb, S. Wolpert, One cannot hear the shape of a drum, Bull. Amer. Math. Soc. (N.S.) 27 (1992), 134-138.

[34] V. Guillemin, Moment maps and combinatorial invariants of Hamiltonian $T^{n}$-spaces, Progress in Math. 122, Birkhäuser, 1994.

[35] V. Guillemin, D. Kazhdan, Some inverse spectral results for negatively curved 2-manifolds, Topology 19 (1980), 301-312.

[36] V. Guillemin, S. Sternberg, Convexity properties of the moment mapping, Invent. Math. 67 (1982), 491-513.

[37] V. Guillemin, S. Sternberg, Geometric quantization and multiplicities of group representations, Invent. Math. 67 (1982), 515-538.

[38] V. Guillemin, S. Sternberg, Symplectic techniques in physics, second ed., Cambridge Univ. Press, 1990.

[39] L. Hörmander, Fourier integral operators. I, Acta Math 127 (1971), 79-183.

[40] A. Iantchenko, J. Sjöstrand, M. Zworski, Birkhoff normal forms in semiclassical inverse problems, Math. Res. Lett. 9 (2002), 337-362.

[41] M. KaC, Can one hear the shape of a drum?, Amer. Math. Monthly 73 (1966), 1-23.

[42] Y. Karshon, L. Kessler, M. Pinsonnault, A compact symplectic four-manifold admits only finitely many inequivalent toric actions, J. Symplectic Geom. 5 (2007), 139-166.

[43] B. Kostant, Orbits, symplectic structures and representation theory, in Proc. U.S.Japan Seminar in Differential Geometry (Kyoto, 1965), Nippon Hyoronsha, Tokyo, 1966, p. 71.

[44] B. Kostant, Quantization and unitary representations. I. Prequantization, in Lectures in modern analysis and applications, III, Springer, 1970, 87-208. Lecture Notes in Math., Vol. 170.

[45] B. Kostant, Symplectic spinors, in Symposia Mathematica, Vol. XIV (Convegno di Geometria Simplettica e Fisica Matematica, INDAM, Rome, 1973), Academic Press, 1974, 139-152.

[46] B. Kostant, On the definition of quantization, in Géométrie symplectique et physique mathématique (Colloq. Internat. CNRS, No. 237, Aix-en-Provence, 1974), Éditions du CNRS, Paris, 1975, 187-210.

[47] B. Kostant, Á. Pelayo, Geometric quantization, a Lie theory approach, to appear as Springer Universitext.

[48] R. De La Llave, A tutorial on KAM theory, in Smooth ergodic theory and its applications (Seattle, WA, 1999), Proc. Sympos. Pure Math. 69, Amer. Math. Soc., 2001, 175-292.

[49] I. Madsen, J. Tornehave, From calculus to cohomology, Cambridge Univ. Press, 1997.

[50] H. P. J. McKean, I. M. Singer, Curvature and the eigenvalues of the Laplacian, J. Differential Geometry 1 (1967), 43-69.

[51] A. Melin, J. Suöstrand, Bohr-Sommerfeld quantization condition for nonselfadjoint operators in dimension 2, Astérisque 284 (2003), 181-244. 
[52] J. Milnor, Eigenvalues of the Laplace operator on certain manifolds, Proc. Nat. Acad. Sci. U.S.A. 51 (1964), 542.

[53] L. Boutet de Monvel, V. Guillemin, The spectral theory of Toeplitz operators, Annals of Math. Studies 99, Princeton Univ. Press, 1981.

[54] B. Osgood, R. Phillips, P. Sarnak, Compact isospectral sets of plane domains, Proc. Nat. Acad. Sci. U.S.A. 85 (1988), 5359-5361.

[55] B. Osgood, R. Phillips, P. Sarnak, Compact isospectral sets of surfaces, J. Funct. Anal. 80 (1988), 212-234.

[56] B. Osgood, R. Phillips, P. Sarnak, Moduli space, heights and isospectral sets of plane domains, Ann. of Math. 129 (1989), 293-362.

[57] Á. Pelayo, S. Vũ Ngọc, Semitoric integrable systems on symplectic 4-manifolds, Invent. Math. 177 (2009), 571-597.

[58] Á. Pelayo, S. Vũ Ngọc, Constructing integrable systems of semitoric type, Acta Math. 206 (2011), 93-125.

[59] Á. Pelayo, S. Vũ Ngọc, Symplectic theory of completely integrable Hamiltonian systems, Bull. Amer. Math. Soc. (N.S.) 48 (2011), 409-455.

[60] Á. Pelayo, S. Vũ Ngọc, First steps in symplectic and spectral theory of integrable systems, Discrete Contin. Dyn. Syst. 32 (2012), 3325-3377.

[61] Á. Pelayo, S. Vũ Ngọc, Hamiltonian dynamics and spectral theory for spinoscillators, Comm. Math. Phys. 309 (2012), 123-154.

[62] J.-M. Souriau, Quantification géométrique, Comm. Math. Phys. 1 (1966), 374-398.

[63] J.-M. Souriau, Structure des systèmes dynamiques, Maîtrise de mathématiques, Dunod, 1970.

[64] T. Sunada, Riemannian coverings and isospectral manifolds, Ann. of Math. 121 (1985), 169-186.

[65] J. A. Tотн, On the quantum expected values of integrable metric forms, J. Differential Geom. 52 (1999), 327-374.

[66] J. A. Tотн, S. Zelditch, Riemannian manifolds with uniformly bounded eigenfunctions, Duke Math. J. 111 (2002), 97-132.

[67] J. A. Tотн, S. Zelditch, $L^{p}$ norms of eigenfunctions in the completely integrable case, Ann. Henri Poincaré 4 (2003), 343-368.

[68] G. M. Tuynman, Quantization: towards a comparison between methods, J. Math. Phys. 28 (1987), 2829-2840.

[69] S. VŨ NGọc, Bohr-Sommerfeld conditions for integrable systems with critical manifolds of focus-focus type, Comm. Pure Appl. Math. 53 (2000), 143-217.

[70] S. VŨ NGọc, Systèmes intégrables semi-classiques : du local au global, Panoramas et Synthèses 22 (2006).

[71] S. VŨ NGọc, Symplectic inverse spectral theory for pseudodifferential operators, in Geometric aspects of analysis and mechanics, Progr. Math. 292, Birkhäuser, 2011, 353-372.

[72] H. WeYL, Über die asymptotische Verteilung der Eigenwerte, Göttinger Nachrichten (1911), 110-117.

$4^{\mathrm{e}}$ SÉRIE - TOME 46 - 2013 - No 5 
[73] H. Weyl, Das asymptotische Verteilungsgesetz der Eigenwerte linearer partieller Differentialgleichungen (mit einer Anwendung auf die Theorie der Hohlraumstrahlung), Math. Ann. 71 (1912), 441-479.

[74] S. Zelditch, Index and dynamics of quantized contact transformations, Ann. Inst. Fourier (Grenoble) 47 (1997), 305-363.

[75] S. Zelditch, Inverse spectral problem for analytic domains. II. $\mathbb{Z}_{2}$-symmetric domains, Ann. of Math. 170 (2009), 205-269.

\author{
Laurent CHARLES \\ Institut de Mathématiques de Jussieu \\ Université Pierre et Marie Curie (Paris VI) \\ Case 247 \\ 4 , place Jussieu \\ F-75252 Paris Cedex 05 \\ E-mail: charles@math.jussieu.fr \\ Álvaro Pelayo \\ School of Mathematics \\ Institute for Advanced Study \\ Einstein Drive \\ Princeton, NJ 08540 USA. \\ and \\ Washington University \\ Mathematics Department \\ One Brookings Drive, Campus Box 1146 \\ St Louis, MO 63130-4899, USA \\ E-mail: apelayo@math.wustl.edu \\ San Vũ NgọC \\ Institut Universitaire de France \\ and \\ Institut de Recherches Mathématiques de Rennes \\ Université de Rennes 1 \\ Campus de Beaulieu \\ F-35042 Rennes cedex, France \\ E-mail: san.vu-ngoc@univ-rennes1.fr
}

